\title{
Channel network concept: an integrated approach to visualize solute transport in fractured rocks
}

\author{
Pirouz Shahkarami ${ }^{1}$ (D) Ivars Neretnieks $^{1} \cdot$ Luis Moreno $^{1} \cdot$ Longcheng Liu $^{1}$ \\ Received: 6 September 2017 / Accepted: 15 August 2018 / Published online: 28 August 2018 \\ (C) The Author(s) 2018
}

\begin{abstract}
The advection-dispersion equation, ADE, has commonly been used to describe solute transport in fractured rock. However, there is one key question that must be addressed before the mathematical form of the so-called Fickian dispersion that underlies the $\mathrm{ADE}$ takes on physical meaning in fractures. What is the required travel distance, or travel time, before the Fickian condition is met and the ADE becomes physically reasonable? A simple theory is presented to address this question in tapered channels. It is shown that spreading of solute under forced-gradient flow conditions is mostly dominated by advective mechanisms. Nevertheless, the ADE might be valid under natural flow conditions. Furthermore, several concerns are raised in this paper with regard to using the concept of a field-scale matrix diffusion coefficient in fractured rocks. The concerns are mainly directed toward uncertainties and potential bias involved in finding the continuum model parameters. It is illustrated that good curve fitting does not ensure the physical reasonability of the model parameters. It is suggested that it is feasible and adequate to describe flow and transport in fractured rocks as taking place in three-dimensional networks of channels, as embodied in the channel network concept. It is argued that this conceptualization provides a convenient framework to capture the impacts of spatial heterogeneities in fractured rocks and can accommodate the physical mechanisms underlying the behavior of solute transport in fractures. All these issues are discussed in relation to analyzing and predicting actual tracer tests in fractured crystalline rocks.
\end{abstract}

Keywords Solute transport $\cdot$ Channel network concept $\cdot$ Non-Fickian behavior $\cdot$ Advection-dispersion equation $\cdot$ Matrix diffusion

\section{Introduction}

In the radioactive-waste-management community, particular effort has been directed toward studying the hydrodynamic dispersion and residence-time distribution of solutes in fractured systems. In this regard, continuum models, i.e., Darcy's law and the advection-dispersion equation, $\mathrm{ADE}$, have been commonly used to describe fluid flow and solute transport in fractured rocks, respectively (Tang et al. 1981; Sudicky and Frind 1982; Małoszewski and Zuber 1985; Hodgkinson and

Electronic supplementary material The online version of this article (https://doi.org/10.1007/s10040-018-1855-6) contains supplementary material, which is available to authorized users.

Pirouz Shahkarami

pirouzs@kth.se

1 Department of Chemical Engineering, Royal Institute of Technology, Stockholm, Sweden
Maul 1988). Essentially, in the classical ADE models, the solute transport acts as if the hydrodynamic dispersion, in its widest sense, behaves similarly to a Fickian-diffusion process that can be characterized by a constant dispersion coefficient, independent of travel distance. In a similar manner, the effect of matrix diffusion has been commonly included in the continuum model by adding a sink term to the ADE, so it can describe the diffusive mass exchange between fractures and the rock matrix by a constant diffusion coefficient (Neretnieks et al. 1982; Tsang 1995; Carrera et al. 1998; Simunek and van Genuchten 2008).

Even though the classical ADE has been shown to be applicable in some homogeneous porous media such as a carefully designed packed bed reactor (Delgado 2006), there are numerous studies suggesting that the continuum model does not adequately represent the real nature of solute transport in geological formations, which often exhibit non-Fickian behavior. (Matheron and de Marsily 1980; Long et al. 1982; Pankow et al. 1986; Berkowitz et al. 1988; McKay et al. 1993; Guerin and Billaux 1994). In a survey study, Gelhar 
et al. (1992) compiled dispersivity observations from 59 different sites and demonstrated that the dispersion coefficient increased with the observation distance. This scale dependency is a clear violation of the ADE assumption, namely that the dispersion coefficient is a fixed property of the porous medium. Many other studies have also reported on the limitation of the $\mathrm{ADE}$ in aquifers containing connected and highly conductive channels (Neretnieks 2002; Becker and Shapiro 2003) as well as in fractured rocks exhibiting anomalous behavior (Berkowitz et al. 2002; Gao et al. 2009; Kang et al. 2015).

Nevertheless, many studies that predict the downstream spreading of the solute lump all the dispersion effects caused by, e.g., velocity variation, into the parameter "macro dispersion coefficient", and solve the ADE assuming, contrary to the field observations, that the transport is Fickian. However, they often fail to address the key question, namely, what is, if ever, the required travel distance or travel time before the socalled Fickian condition is reached and the ADE becomes physically reasonable.

Furthermore, when the continuum model is employed to interpret field tracer tests, it has often been found that much larger matrix diffusion coefficients than laboratory measurements are required to reproduce the observed tracer breakthrough curves, BTCs, indicating that matrix diffusion in the field would be enhanced in some way. In literature, this enhanced coefficient is often referred to as the "field-scale matrix diffusion coefficient" (Liu et al. 2007). In a survey study, Zhou et al. (2007) reviewed 40 field tracer tests conducted at different fractured sites and analyzed the tracer BTCs by the continuum model. They noted that the field-scale matrix diffusion coefficients generally exhibited enhanced values in comparison to laboratory measurements and that the coefficients increased with observation distance. Similar results were also found in other studies suggesting that the laboratory-scale matrix diffusion coefficient may be orders of magnitude smaller than the so-called field-scale matrix diffusion coefficient (Becker and Shapiro 2000; Shapiro 2001; Neretnieks 2002; Liu et al. 2003).

In literature, the enhanced matrix diffusion effect in naturally heterogeneous fractured media is attributed to several physical mechanisms at different scales. These include, but are not limited to, diffusion into fracture in-fillings (Neretnieks 2002), advective transport between fast and slow pathways (Shapiro 2001), diffusion into the stagnant water zones (Neretnieks 2005; Shahkarami et al. 2015), and fractal structure of transport paths (Liu et al. 2007). It is also well recognized that the impacts of the various mechanisms mentioned above, together with that of matrix diffusion, are strongly influenced by flow conditions in the transport paths. However, the flow condition cannot, in most cases, be adequately approximated by the continuum model, given the fact that the heterogeneities, including aperture variability, cannot be captured.

Based on the aforementioned considerations, it is believed that the overwhelming efforts directed toward defining the effective entities to describe the transport problem in fractured rock are misplaced for the detailed understanding of hydraulic behavior and solute transport mechanisms. Obviously, there are important processes that the continuum model does not capture well, especially when flow in fractured rocks takes place in many pathways that do not, or very little, mix with water in other pathways. Not to mention that estimation of the effective entities is subject to inherent biases and errors.

While aware of other reasonable approaches (Haggerty and Gorelick 1995; Berkowitz and Scher 1997; Neuman et al. 1987; Cushman and Ginn 2000; Becker and Shapiro 2003), it is suggested that it is feasible and more physically meaningful to describe flow and solute transport in fractured rocks as taking place in three-dimensional (3D) networks of channels, as embodied in the channel network concept, CN-concept (Moreno and Neretnieks 1993). In this paper, various phenomena that give rise to dispersion in channels in fractures are briefly discussed. It is followed by studying the Taylor dispersion in tapered channels, which are deemed to better describe flow paths in fractured crystalline rocks (Liu et al. 2017). Following that, by some simple calculations, it is illustrated that man-made field experiments that are commonly used to assess dispersion properties are subject to inherent difficulties because in experiments with larger flow rates, dispersion can be dominated by other mechanisms than what will prevail under natural flow conditions. To highlight the differences between the continuum model and the $\mathrm{CN}$-concept, the channel network model, CNM, and its computer code, CHAN3D, are used to study the converging tracer test STT-1, part of the TRUE-1 project (Elert and Svensson 2001). The CNM is founded on the CN-concept and is, in fact, the CHAN3D implementation of the $\mathrm{CN}$-concept, which can further be evolved to include additional mechanisms and geometric features.

One contribution of this paper is, therefore, to study the Taylor dispersion in tapered channels and find the time threshold beyond which the advection-dispersion equation is applicable in individual channels. The second contribution of this paper is to present a comparative analysis and highlight the difference between the dispersions expected under forced- and natural-gradient flow conditions. The third contribution is to present arguments and examples from actual tracer tests to highlight the uncertainties and ambiguities regarding the use of the effective entities to interpret field experiment results. The fourth contribution is to demonstrate the applicability of the $\mathrm{CN}$-concept to capture the effect of spatial heterogeneities and to visualize contaminant transport in fractured rocks.

\section{The channel-network-transport model in fractured rocks}

Crystalline rocks contain systems of intersecting fractures whose apertures vary from fully closed to usually less than a 
millimeter (Moreno et al. 1990). The more open locations in a fracture may connect over long distances and form potential pathways through which water can flow in the presence of hydraulic gradients. The flow paths in the individual fracture are called channels, fully recognizing that hydraulic gradients applied in other directions will generate other flow paths. Within a rock volume, when the stochastically oriented fractures intersect, the multitude of channels can connect and form a 3D network of channels. The water flow rates and velocities differ between channels and the velocity may also vary across the width of individual channels because the aperture varies across the channels. They are tapered. At channel intersections where waters from two or more channels meet, the waters may mix to varying degrees, and solute in the flowing waters may move partly into one and partly into another channel. At the intersections, the solute can, therefore, be divided up into an enormous number of pathways, composed of a series of channels. In each channel, the solute transport can be retarded by matrix diffusion, diffusion into stagnant water zones, and sorption, as well as be affected by mixing between the streamlines in the channel. It should further be noted that when water flows in a narrow channel, the matrix diffusion would increasingly become radial as the penetration length by diffusion becomes larger than the channel width, thus enhancing the rate of mass exchange with the water in the porous rock matrix and causes increased retardation (Neretnieks 2017). These physical mechanisms underlying the natural behavior of solute transport in fractured rocks are incorporated in the CNM. Flow rate distribution is primarily determined by solving the Darcy flow in each channel in the network. By a time-domain particle tracking method (Tsang and Tsang 2001) a multitude of pathways is then generated in the rock volume that the particles traverse from the injection point through the network to an observation point. Because excessively high computational demand is required to evaluate the mixing at the $3 \mathrm{D}$ channel intersections, the model assumes that the mixing is complete. The immediate implication of this assumption is that the probability of solute particles in the inflow channel to enter the outflow channels is proportional to the channel outflow rates. In this way, solute particles can traverse different transport paths and their residence times can be determined in channels constituting the pathways. It is also possible to determine where the solute will arrive and how the concentration will vary over time in the pathways.

In what follows, dispersion is studied in scenarios when samples from different pathways are collected and mixed, e.g., in a converging tracer test. The main objective of this section is to demonstrate how the CNM can be applied to capture the effect of varying velocity field inherent in shortrange field tracer tests. In the following section, it is also discussed how the use of the ADE and the macro dispersion coefficient can be misleading when applied to predict tracer test results.

\section{The STT-1 test: estimation of flow porosity from hydraulic data}

In this part, a predictive modeling of the converging tracer test STT-1 is performed using the non-sorbing tracers Uranine and tritiated water, HTO (Elert and Svensson 2001). The tracers are chosen to characterize the residence time distribution of water as they experience negligible interaction with the rock matrix. The STT-1 test is well defined and the corresponding data, including its statistical properties are analyzed and well understood. The test was made at about $400 \mathrm{~m}$ depth in a fractured rock surrounding a more prominent waterconducting fracture, the so-called "feature A". The tracers were injected over $4 \mathrm{~h}$, as shown in Fig. 1, and the collection took place at about $5 \mathrm{~m}$ distance from the injection point (Elert and Svensson 2001). The flow rate was induced by pumping water from the collection point at a rate of $0.024 \mathrm{~m}^{3} / \mathrm{h}$ to achieve a converging flow field.

To simulate the experiment, the tracer transport is modeled in a rock volume of $20 \mathrm{~m} \times 20 \mathrm{~m} \times 20 \mathrm{~m}$. It is assumed that the feature $\mathrm{A}$ and a large number of smaller fractures around it are parts of a 3D network of channels and that their conductivities can be characterized by a single statistical distribution. The hydraulic and structural properties of channels are generated based on the detailed measurements and analysis performed in the Äspö Hard Rock Laboratory, HRL, in Sweden (Winberg et al. 2000; Shahkarami et al. 2016). Most importantly, the average channel length is set to $0.5 \mathrm{~m}$ and the channel transmissivities are assigned randomly from a lognormal distribution with mean $\mu=-7.8$ and standard deviation $\sigma=0.97$, as given in Fig. S1 of the electronic supplementary material (ESM). These data are derived from hydraulic measurements in boreholes intersecting the rock within and surrounding the test volume (Neretnieks and Moreno 2003).

The channel aperture can be estimated from transmissivity using the following equation for the transmissivity of a single channel (Maloszewski and Zuber 1993)

$\operatorname{Tr}=3.8 \times 10^{6} \times b^{3}$

where $\operatorname{Tr}$ is the channel transmissivity in $\mathrm{m}^{2} / \mathrm{s}$ and $b$ is the channel half-aperture in meters. Hence, the half-aperture distribution in the system can be found, as shown in Fig. S2 of the ESM. Furthermore, from the distribution, one can estimate the average half-aperture of the ensemble of the channels, $b_{\text {avg }}$. The subscript $m$ denotes the mean value over of the ensemble of the channels. This can be obtained by the following equation that gives $b_{\text {avg }}=2.6 \times 10^{-5}$.

$b_{\text {avg }}=\int_{0}^{\infty} b \times p(b) d b$

$p(b)$ is the probability density function of the channel halfaperture which follows a lognormal distribution, Fig. S2 of the ESM. 
Fig. 1 Injection curves for Uranine and tritiated water (HTO)

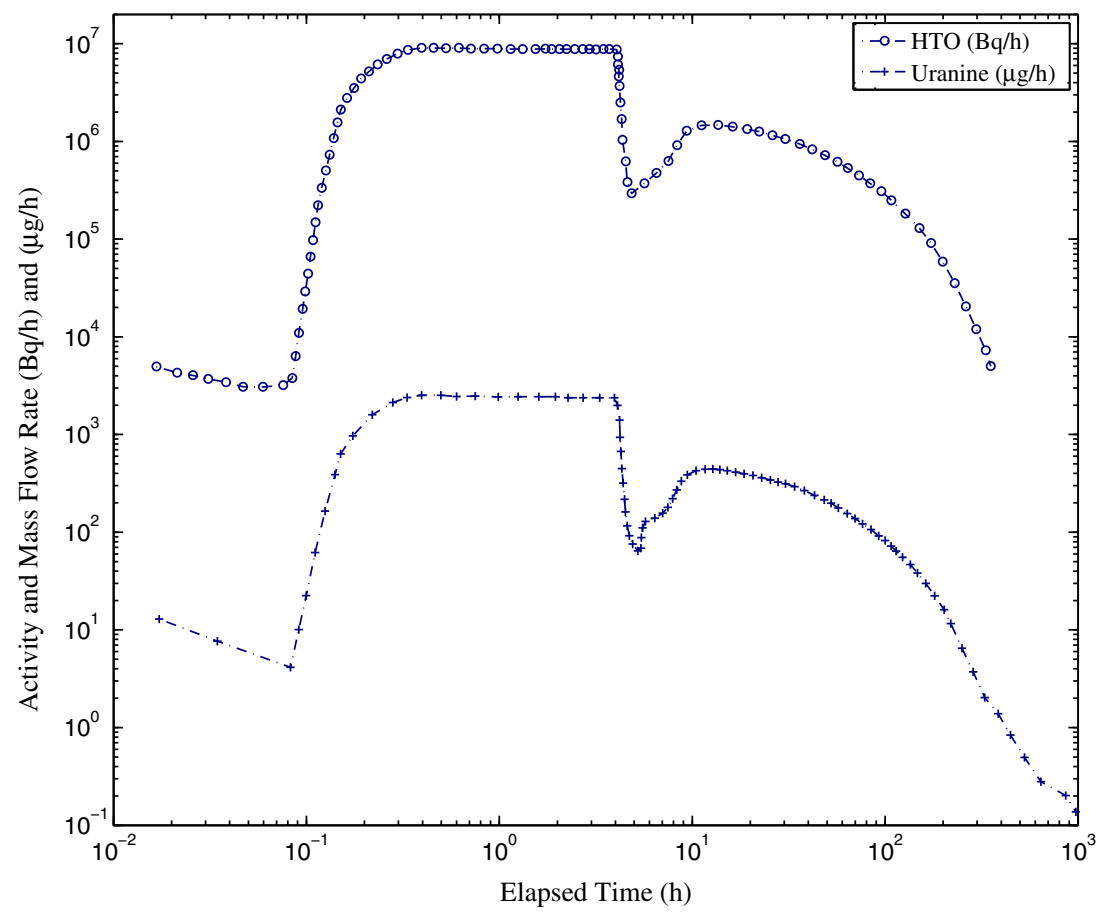

For the 3D flow structure, the water flows in a radially converging direction within the spherical volume-enclosing feature A. It can be shown that the flow porosity, $\phi$, is related to the average channel half-aperture, $b_{\text {avg }}$, and the specific flow-wetted-surface area, $\alpha_{R}$, by (Shahkarami et al. 2016)

$\phi=b_{\text {avg }} \alpha_{\mathrm{R}}$

With the estimated $\alpha_{\mathrm{R}}=10 \mathrm{~m}^{2} / \mathrm{m}^{3}$ for the STT- 1 test, this gives $\phi=2.6 \times 10^{-4}$. These values together with the transmissivity distribution data are used to generate the channel network.

\section{The STT-1 test: simulation of the non-sorbing tracers transport}

A thousand particles are injected into the network and collections take place at the observation point at $5 \mathrm{~m}$ distance from the source. Every particle is advected through a sequence of different channels with different flow rates resulting in a distinct residence time. In this way, it is possible to consider the impact of collecting and mixing of the tracers, which have experienced different residence time and have been subject to different spreading mechanisms along the pathways to the collection point. This feature is in line with the concept of velocity dispersion (Neretnieks 1983) and is embodied in the CNM. The particles' travel-time histogram distributions for one channel-network realization are shown in Fig. 2.

In the following simulations, a hundred realizations of the channel network are made to represent the stochastic nature of the flow distribution in the rock volume. The only active spreading mechanisms in individual channels are advection and matrix diffusion. Negligible Fickian-like dispersion is expected in channels, as is discussed in the following section. The simulation results for the two selected tracers, i.e., Uranine and HTO, are shown in Figs. 3 and 4, respectively. The BTCs are shown as probability density maps, composite of a hundred realizations, and the mean of the resulted BTCs are shown as the solid lines. For comparison purpose, the experimental BTCs of Uranine and HTO are also included in the figure.

The results suggest that the CNM achieves very good predictions of the tracers' BTCs both at the rising limb and the tailed part of the curves. The tailing, in the absence of other dispersive mechanisms, is commonly cited as the apparent consequence of matrix diffusion (Grisak and Pickens 1980; Neretnieks et al. 1982; Maloszewski and Zuber 1993; McKenna and Meigs 2000); however, in this experiment the tailing slope is greater (steeper) than the well-known " $-3 / 2$ double-log slope" for matrix diffusion (Haggerty et al. 2000). Possible explanations could be that the asymptotic tail of the injection curves or velocity variation between different pathways led to the observed tailed distribution. In fact, measurements in the vicinity of feature A have revealed that transmissivities vary over more than four orders of magnitude, which can yield paths of fast and slow flow. Therefore, paths with a fast flow result in rapid, early breakthrough of tracers, and those travelling along slower paths, on the other hand, experience greater retardation by matrix diffusion. 
Fig. 2 Travel time histogram distributions for one channelnetwork realization (number of injected particles $=1,000$ )

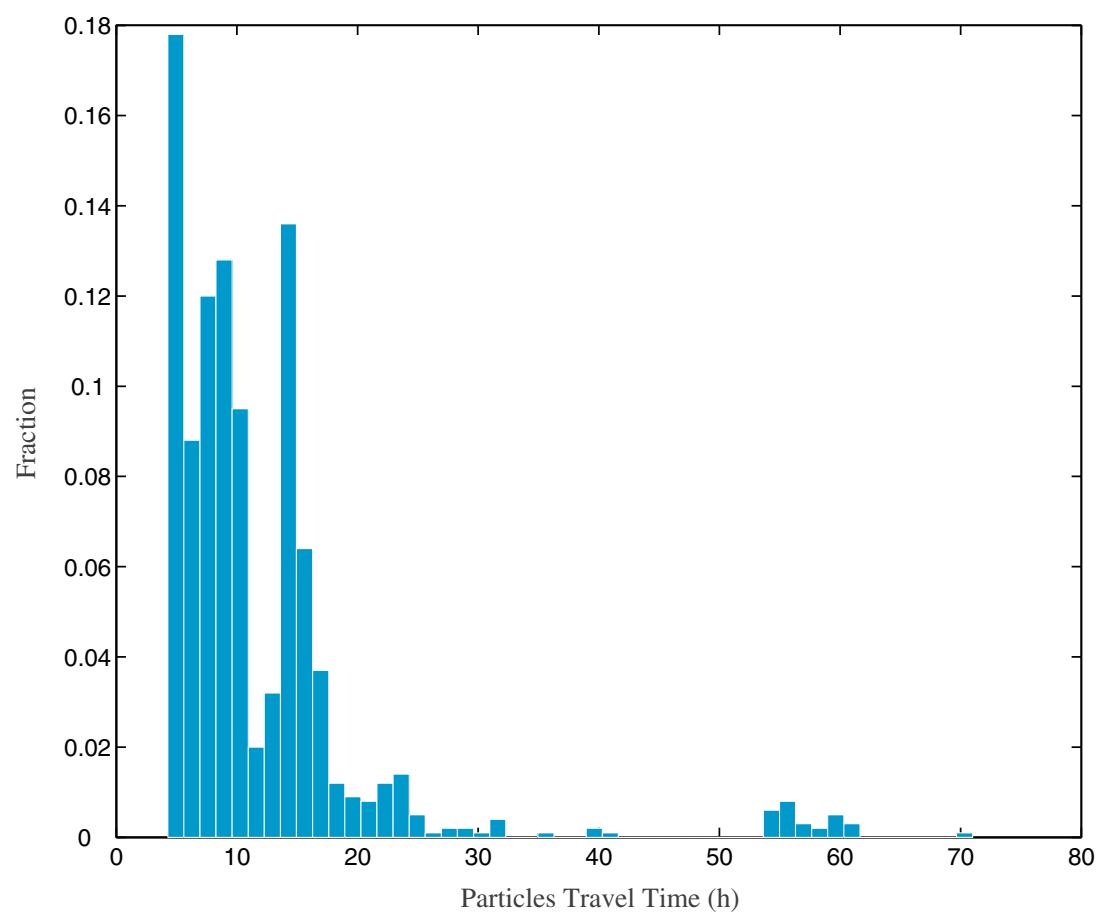

For comparison purpose, and also to point out the relative contribution of matrix diffusion, the resulted mean BTCs for Uranine and HTO are normalized relative to their maximum values. New simulations are also made under the same injection and flow conditions as in the original test; however, this time, given two imaginary tracers with smaller values of matrix diffusion coefficient, called tracer A and tracer B. These tracers are given three orders of magnitude smaller matrix diffusion coefficients compared to those of Uranine and HTO, respectively. The predicted results are plotted in Fig. 5 for all the tracers.

It can be seen that the normalized BTCs for the real tracers with two different diffusion coefficients, $\mathrm{De}_{U}=7.6 \times$ $10^{-14} \mathrm{~m}^{2} / \mathrm{s}$, De $e_{\text {HTO }}=1.2 \times 10^{-13} \mathrm{~m}^{2} / \mathrm{s}$, are practically identical and exhibit similar tailings. Furthermore, the BTCs of the imaginary tracers with relatively small values of matrix
Fig. 3 Simulated and experimental breakthrough curves (BTCs) for Uranine

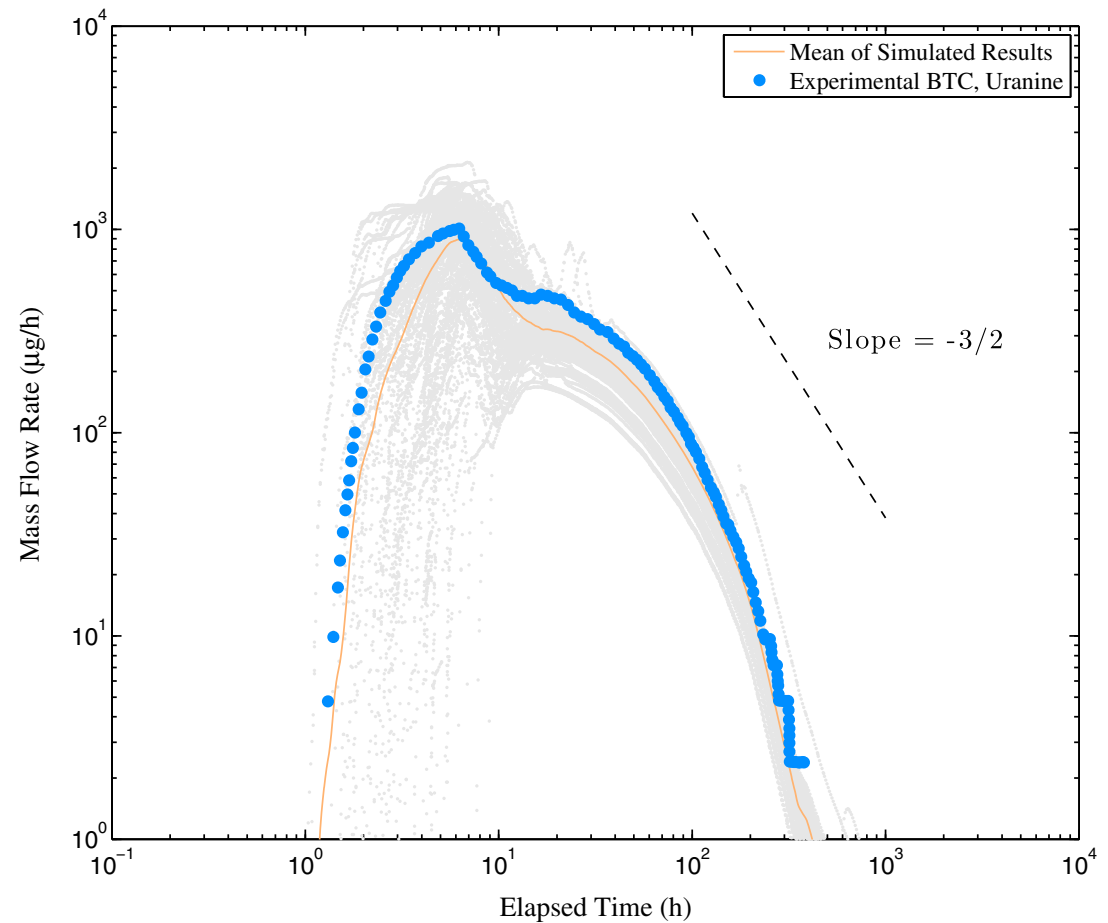


Fig. 4 Simulated and experimental BTCs for HTO

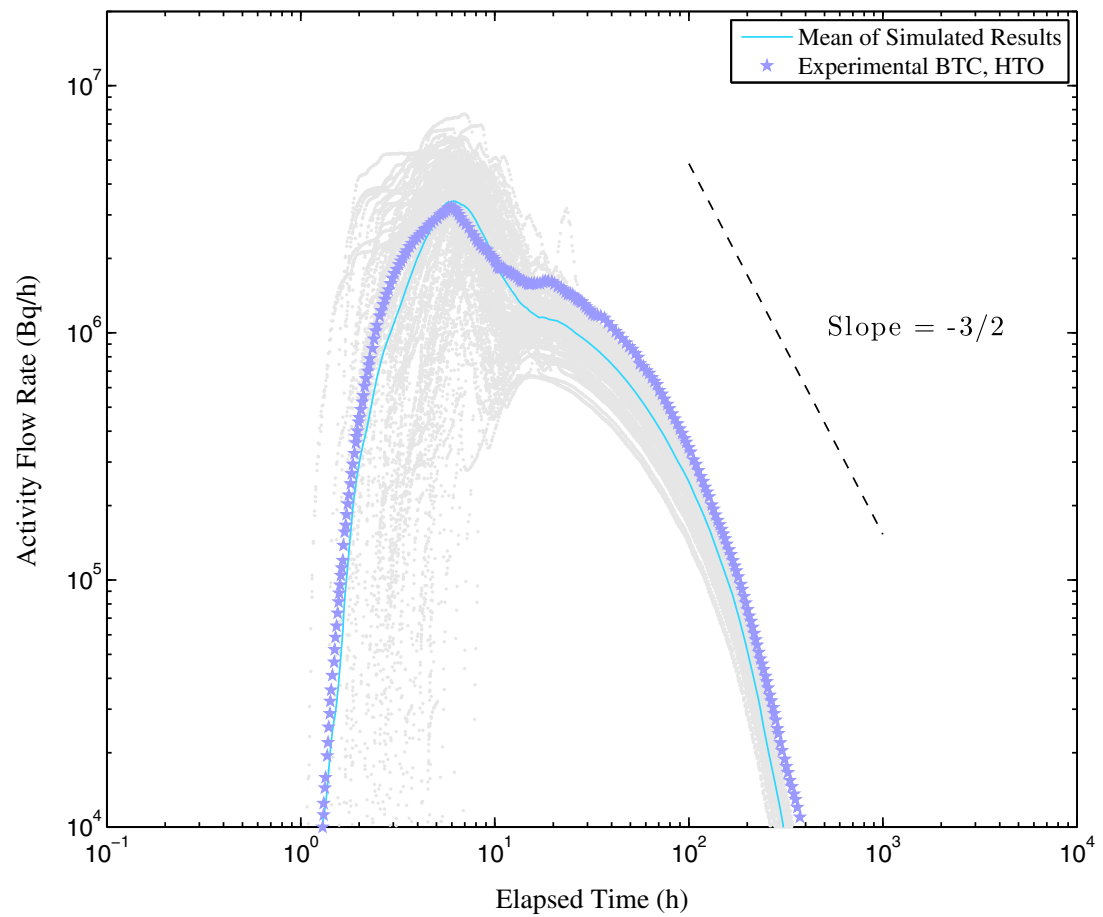

diffusion coefficients, exhibit strong tailings and, essentially, very weak separations are observed between the BTCs of the injected tracers. These findings suggest that matrix diffusion has marginal effect on the BTC tailings for these non-sorbing tracers in this experiment.

The second test explores the effect of velocity distribution in the network. For this purpose, new simulations are made assuming that all the channels in the network have similar transmissivities, i.e., $\mu=-7.8$ and $\sigma=0$. Thus, contrary to the original case where flow channeling results in different particle residence times, most of the particles traverse along a single pathway with a fixed residence time. The simulation results are shown and compared with the original case in Fig. 6.

The predicted BTCs with $\sigma=0$ feature: (1) delayed early arrival of the tracers, (2) a very sharp rising limb, and (3) less
Fig. 5 Normalized BTCs for tracers with different molecular diffusion coefficients

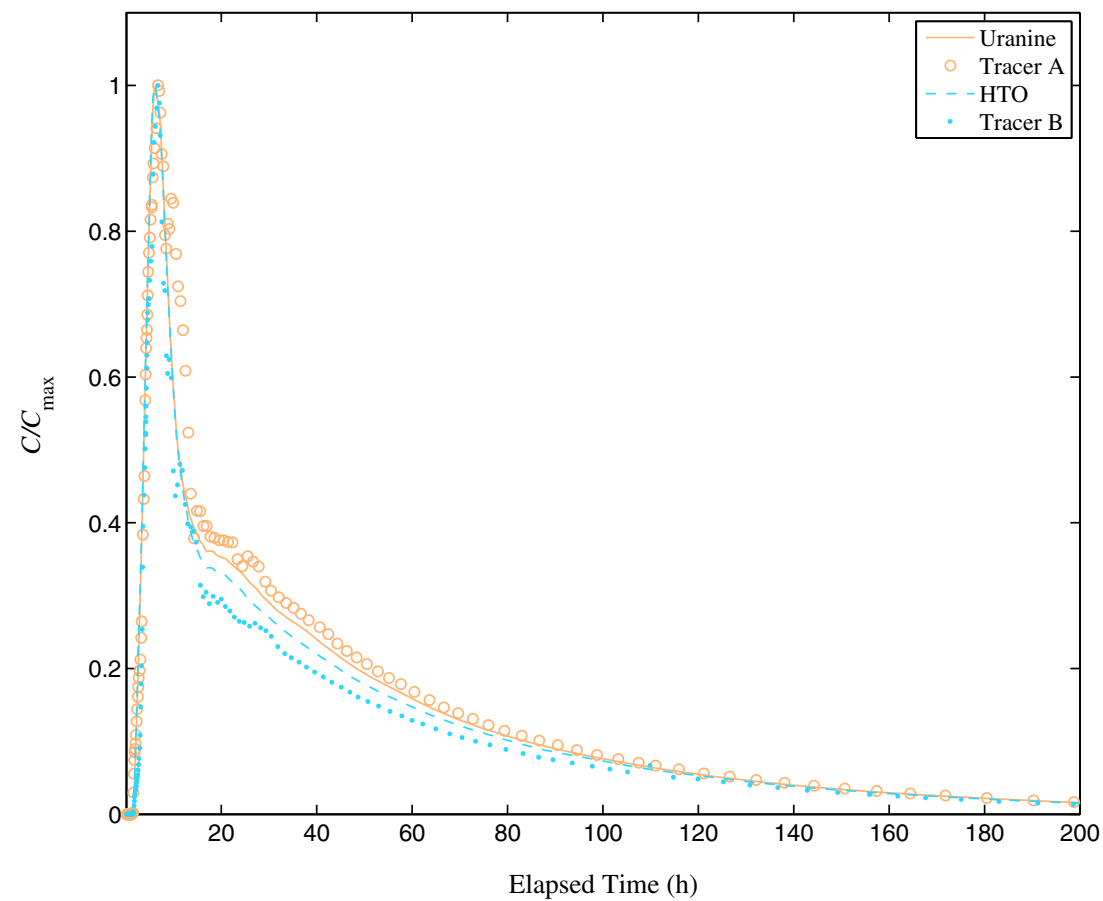


tracer mass recovery. The results suggest that the velocity variation can largely affect the early-time behavior of the tracers; therefore, a single pathway cannot solely explain the tracer transport behavior in the test rock volume. However, under the STT1 test conditions, the tracer latetime behaviors remain practically unaffected and the simulated BTCs exhibit strong tailings similar to those observed in the experimental curves. In fact, closer inspection of the simulated BTCs, when $\sigma=0$, reveals that the outlet concentrations preserve the shapes of the injection curves. That is, the relatively steep tailing observed in the BTCs (slope $>-3 / 2$ ) is controlled by the injection curves given in Fig. 1; therefore, it can be inferred that the overall dispersion observed in the BTCs are mainly controlled by the injection curves as well as velocity distribution within the network.

It is worth emphasizing that the dispersion caused by flow channeling in a rock volume is shown to increase in proportion to the observation distance, due primarily to the effect of velocity dispersion (Liu et al. 2017). This is embodied in the $\mathrm{CNM}$; therefore, in more general scenarios, where essential spatial heterogeneities in fractured rocks, i.e., deterministic and stochastic fractures, fracture zones and tunnels, are introduced into the system, the CNM can explain the apparent scale dependency of dispersion, provided conductive channels connect to form effective transport paths with different flow rates.

\section{Use of the Taylor dispersion and continuum model in the STT-1 test}

In the preceding simulations, hydrodynamic dispersion in the channels is neglected because the individual channels are assumed to have constant aperture and width. In tapered channels, the only expected dispersion is the Taylor dispersion across the channel width, which may contribute to the longitudinal dispersion under the conditions of the experiment. In this section, it is explored when it is appropriate to consider the Taylor dispersion in a tapered channel. It is worth emphasizing that when Taylor dispersion is fully developed, the dispersion coefficient becomes constant and the ADE is valid; furthermore, the effects of changes in channel width and flow condition on the Taylor dispersion is studied in tapered channels. It is also shown that forced-gradient experiments can result in considerably different dispersion than what can apply in the natural-gradient case. For these purposes, the data evaluated for the tracer test STT-1 are incorporated in the continuum model to examine whether it can correctly be used to interpret the test results for the nonsorbing tracers.

\section{Taylor dispersion in tapered channels}

Taylor (1953) and Aris (1956) argued that in a circular tube with radius $R$ where the velocity profile is parabolic, the spreading of a tracer pulse propagates similarly to a Gaussian pulse, provided the time of interest is sufficiently long. That is, the pulse traveling with the mean fluid velocity, $U$, becomes broader proportionally to the square root of the travel distance - exactly as a pulse would behave with a larger molecular diffusion coefficient $\left(D_{\mathrm{w}}\right)$. It is shown that the axial dispersion of the tracer in the tube can be described by a constant dispersion coefficient $\left(D_{\mathrm{L}}\right)$ given by

$D_{\mathrm{L}}=D_{\mathrm{w}}+\frac{U^{2} R^{2}}{48 \times D_{\mathrm{w}}}$

Liu et al. (2017) studied the solute transverse mixing along a tapered channel, as depicted in Fig. 7, and extended the application of the Taylor dispersion to noncircular channels. They derived an identical expression to the Taylor-Aris dispersion coefficient, provided that the channel aperture $2 b_{\mathrm{f}}$ is much smaller than the channel width $2 W_{\mathrm{f}}$. The only difference was that the tube radius in Eq. (4) was replaced by half-width of the channel to give

$D_{\mathrm{L}}=D_{\mathrm{w}}+\frac{U^{2} W_{\mathrm{f}}^{2}}{48 \times D_{\mathrm{w}}}$

However, as mentioned earlier, the preceding equations are valid only if the required conditions to use the Taylor dispersion are met in the both geometries. A simple theory is presented in the Appendix to examine the required condition for the appearance of Fickian dispersion in a tapered channel. It is shown that the travel time must be equal to or greater than 0.6 of the characteristic time of diffusion across the channel width. In mathematical terms it can be written

$T=\frac{D_{\mathrm{w}} L}{U W_{\mathrm{f}}^{2}} \geq 0.6$

where $T$ is the dimensionless characteristic diffusion time. For the purposes of the present study, the region $0.02<T<0.6$ is considered to be the transition region between when advective transport prevails and when Taylor dispersion does. The Peclet number with respect to the channel length can also be expressed as,

$\mathrm{Pe}=\frac{U L}{D_{\mathrm{L}}} \cong 48 \times \frac{D_{\mathrm{w}} L}{U W_{\mathrm{f}}^{2}}=\frac{\text { Axial diffusion time scale }}{\text { Axial advection time scale }}$

This Peclet number characterizes the ratio of advective transport rate to the rate of dispersive transport and is indicative of the degree of mixing in the channel. Here, again, one 
Fig. 6 Effect of velocity distribution and injection function on BTCs

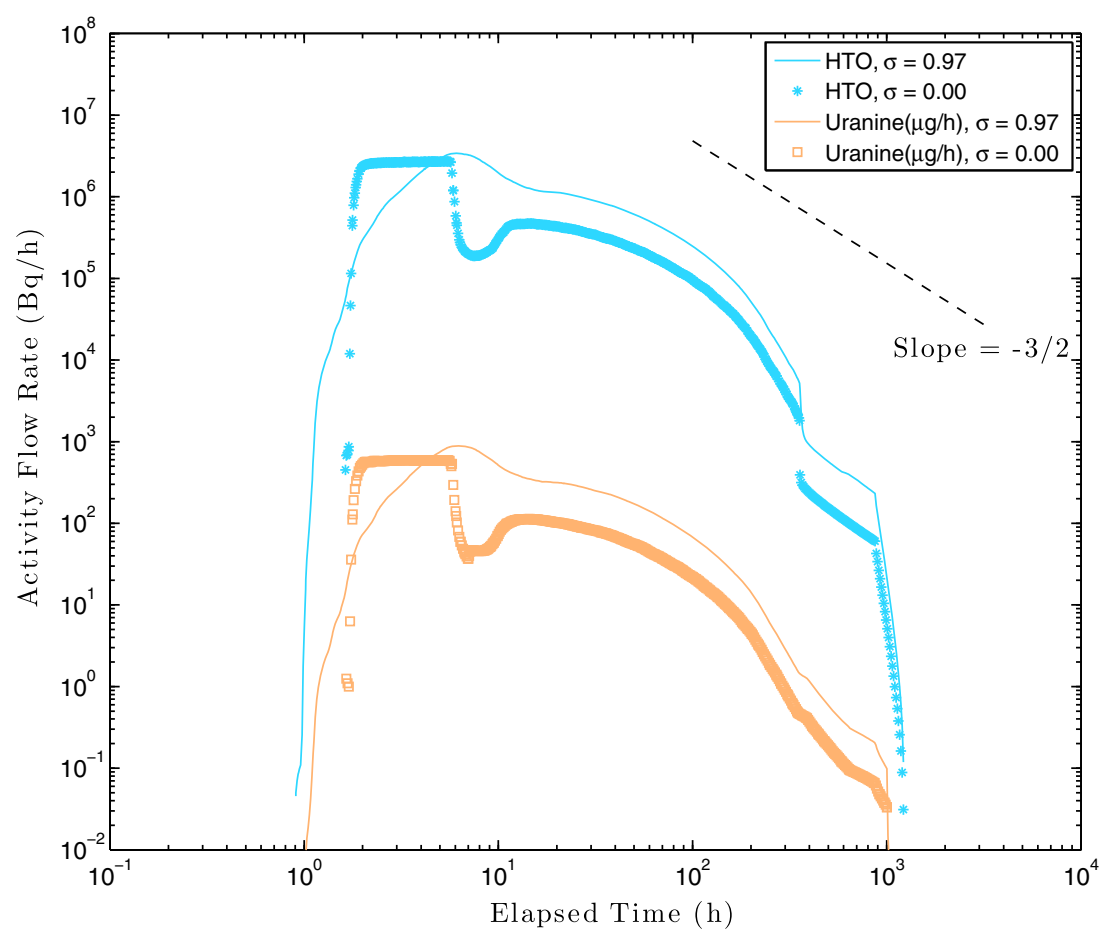

may note that Eqs. (5) and (7) are valid only if the required condition to use the Taylor dispersion is met.

\section{Is the ADE valid under the STT-1 test conditions?}

Following the aforementioned considerations, the effects of changes in the channel width and flow condition on the dispersion are explored under the conditions of the STT-1 test (Table 1). Firstly, it is assumed here that the time of interest is sufficiently long to allow the diffusion across the tapered channel to effectively even out the concentration over the channel width. In other words, the Taylor dispersion concept and ADE in each channel are assumed applicable.

For illustration purpose, channel lengths are set to vary between 0.1 and $5 \mathrm{~m}$ and the widths are set to range from 0.01 to $0.1 \mathrm{~m}$. These values are within the ranges observed in Swedish rock masses at depths below $100 \mathrm{~m}$ (Tsang and Neretnieks 1998). With these data and for a channel length and width of, respectively, 1 and $0.1 \mathrm{~m}$, the Peclet number in a channel becomes

$\mathrm{Pe} \cong 48 \times \frac{D_{\mathrm{w}} L}{U W_{\mathrm{f}}^{2}}=0.117$

However, this requires that the dimensionless characteristics diffusion time equals $T=0.0024$ which is much smaller than $T=0.6$, which suggests that negligible mixing between streamlines is expected for the preceding 1-m-long channel in this study. Therefore, the velocity-induced dispersion in the channel, i.e., the mass-spread expected because solute particles travel with different advection times induced by the parabolic shape of the velocity profile, prevails and the ADE cannot be used in this case. If the ADE were valid, the low
Fig. 7 Cross section of a tapered channel in a fracture plane. $b_{0}$ is the largest aperture of the tapered channel located at $y=W_{\mathrm{f}}$

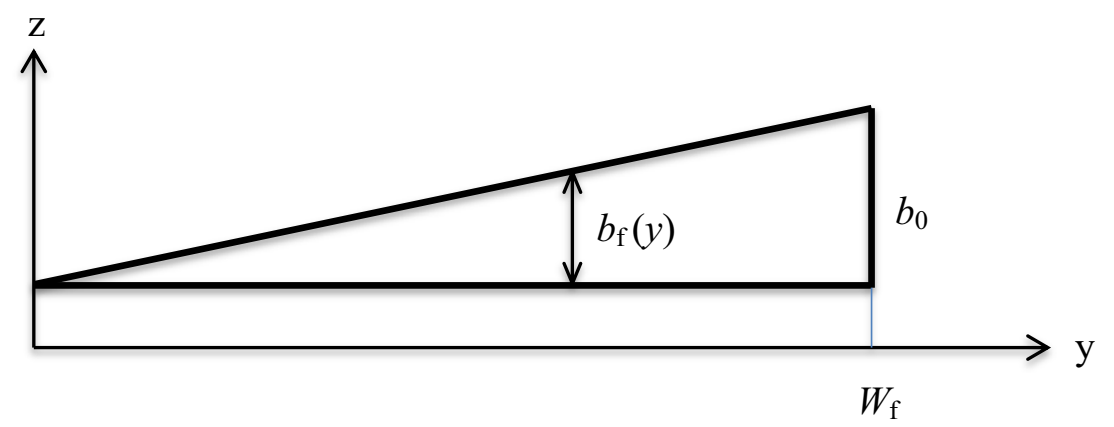


Table 1 The data evaluated for the tracer test STT-1 after (Shahkarami et al. 2016)

\begin{tabular}{ll}
\hline Entity & Value \\
\hline Mean residence time, $t_{\mathrm{m}}(\mathrm{h})$ & 34 \\
Pumping flow rate, $Q\left(\mathrm{~m}^{3} / \mathrm{h}\right)$ & 0.024 \\
Mean velocity, $U(\mathrm{~m} / \mathrm{s})$ & $4.08 \times 10^{-5}$ \\
Diffusivity in water, $D_{\mathrm{w}}\left(\mathrm{m}^{2} / \mathrm{s}\right)$ & $1 \times 10^{-9}$ \\
Specific flow-wetted-surface area, $\alpha_{\mathrm{R}}\left(\mathrm{m}^{2} / \mathrm{m}^{3}\right)$ & 10 \\
Flow porosity & $2.6 \times 10^{-4}$ \\
\hline
\end{tabular}

estimated value of the Peclet number compared to $\mathrm{Pe}=1$ would suggest that dispersion strongly dominates over advection in determining the residence time distribution of the tracer (Huysmans and Dassargues 2005). In other words, the advection term could be neglected to obtain the BTC. Even if in the experiment the entire flow path were to consist of one channel, i.e., $L=5 \mathrm{~m}$, the Peclet number would equal $\mathrm{Pe}=0.59$; however, the use of ADE is valid only if the required condition in Eq. (6) is met. Even for the full length of the flow path in the experiment, i.e., $L=5 \mathrm{~m}$, Taylor dispersion does not develop in a 0.1 -m-wide channel $(T=0.012)$, whereas in a $0.01-\mathrm{m}$ wide channel $(T=1.25)$ it would.

Contrary to the tracer test experiment, the situation is quite different under natural-gradient condition where water flows with lower velocity. With a gradient of $0.003 \mathrm{~m} / \mathrm{m}$, and mean transmissivity of $1 \times 10^{-7.8} \mathrm{~m}^{2} / \mathrm{s}$, the mean velocity under natural-gradient condition is $U=1.52 \times 10^{-7} \mathrm{~m} / \mathrm{s}$. Thus, for a channel with $L=1 \mathrm{~m}$ and $W_{\mathrm{f}}=0.1 \mathrm{~m}$, the Peclet number and dimensionless diffusion time, respectively, becomes $\mathrm{Pe}=31.6$ and $T=0.67$.

This implies that under the natural-gradient condition, the Fickian condition may be satisfied and the ADE may become valid in individual channels, which is in contrast to the field experiment where the spreading of the tracer pulse is caused by (1) velocity-induced dispersion in individual channels, i.e., advection, and (2) separation of the advective pathways, i.e., flow channeling. These results indicate that forced-gradient experiments can result in considerably different dispersion than what can apply in the natural-gradient case for the typical channel widths. One can then conclude that this is another reason why the validity and usefulness of the ADE needs to be carefully investigated before being employed to interpret the tracer test results and why use of the macro dispersion coefficient may be misleading.

To further address this issue, an attempt is made to simulate the STT-1 test with the continuum transport model, which is done by using the ADE, neglecting the matrix diffusion effect, and calibrating the model parameters, Peclet number, Pe, and water mean residence time, $t_{\mathrm{w}}$. As shown in Fig. 8, a very good fit to the tracer test results can be obtained for the $\mathrm{Pe}=5$ and $t_{\mathrm{w}}=5 \mathrm{~h}$. In other words, if the results were interpreted using the continuum model, the inevitable conclusion would be that the dispersion caused by the injection process and heterogeneous velocity field in the rock mass around feature A could solely be described by a large macro dispersion coefficient $D_{\mathrm{L}}=2.78 \times 10^{-4} \mathrm{~m}^{2} / \mathrm{s}$. However, the validity of the ADE and the interpretation of the result, as previously discussed, are questionable under the experiment conditions and the ADE cannot successfully describe the tracer concentration distribution if the test flow conditions are changed, e.g., if the observation distance is increased or flow rate decreased. In other words, one should not feel satisfied with good curve fits of the experimental results unless the model and the parameters used are physically reasonable and have been verified independently.

\section{Rock matrix diffusion at the field scale}

In this section, the focus is placed on the retarding effect of rock matrix diffusion at the field scale. The concern is raised that the field-scale matrix-diffusion coefficient and its value, found from the continuum model, may not correctly characterize the matrix diffusion effect. It is then demonstrated how the CNM can readily be employed to incorporate the heterogeneities in the system and explain the enhanced matrix diffusion effect that is often observed in tracer test experiments, provided the experiment conditions are well defined.

\section{Continuum model and field-scale matrix diffusion coefficient}

Rock matrix is porous and allows solute to diffuse into the micro-pores of the matrix where they can sorb onto the inner surfaces of the matrix and, thus, be withdrawn from the flowing water in a fracture. In this fracture-matrix system, the diffusion coefficient is widely believed to be the key parameter to describe the diffusion process within the matrix as well as the diffusive mass exchange between the fracture and the matrix. It is therefore not surprising to find numerous studies in literature on in-situ measurements of diffusion coefficient in rock samples as well as field tracer tests to find this coefficient (Skagius and Neretnieks 1986; Ohlsson and Neretnieks 1995; Ohlsson et al. 2001; Reimus et al. 2003). The continuum model has commonly been used to estimate the diffusion coefficient from the tracer test BTCs. This is mainly because the model is relatively simple and can easily be implemented. Evidence from a few controlled tracer tests supports the use of the continuum model together with the insitu-measured matrix diffusion coefficients (Maloszewski and Zuber 1990; Moench 1995; Jardine et al. 1999); there are, however, several other studies suggesting that the estimated field-scale matrix diffusion coefficients may be orders of 
Fig. 8 ADE-predicted results and experimental BTC for Uranine

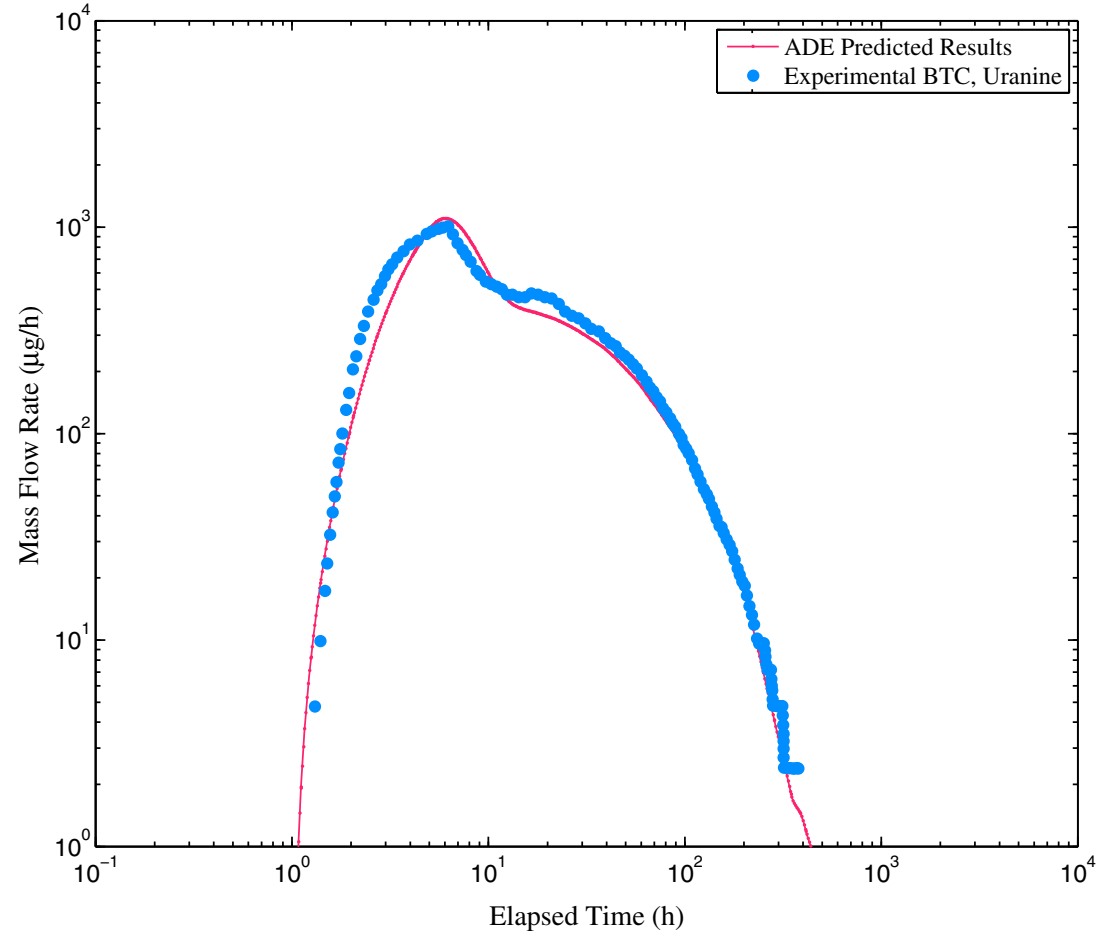

magnitude larger than the values obtained in the laboratory (Zhou et al. 2007).

The predicted enhanced value for the matrix diffusion coefficient is the inevitable consequence of using the continuum model that stems from the inadequacy of the model to capture the effect of spatial heterogeneities in the system. In other words, when the continuum model is used to interpret a field tracer test, the calibrated matrix diffusion coefficient incorporates additional and often complex transport mechanisms that are not considered in the simple model. The coefficient is, therefore, accepted as an effective parameter. Most importantly, the continuum model completely ignores the potential influences of varying the velocity field, as well as the influences of stagnant water zones, STWZs, on the matrix diffusion effect.

To further clarify this issue, the analytical solution of Shahkarami et al. (2015) is used. The solution, Eq. (9), is obtained for solute concentration in a channel, Fig. 9, which is the basic building block of the CNM. The solution allows a clear distinction of transport processes and can be used to analyze the relative contribution of different mechanisms on solute transport retardation. The analytical solution in the Laplace domain is given by

$C_{\mathrm{f}}=C_{\mathrm{f} 0} \times \exp (-\kappa A)$

with

$A=\left[\Omega_{\mathrm{f}}\right]+\left[N \times \sqrt{\Omega_{\mathrm{s}}} \times \tanh \left(2 \sqrt{\Omega_{\mathrm{s}}}\right)\right]$ and

$\kappa=\frac{1}{\frac{1}{2}+\sqrt{\frac{1}{4}+\frac{A}{\mathrm{Pe}}}}$

In Eq. (10), $\Omega_{\mathrm{f}}$ and $\Omega_{\mathrm{s}}$ denote the contribution of the flowing channel and STWZ, respectively. If the rock matrix thickness is large, $\Omega_{\mathrm{f}}$ and $\Omega_{\mathrm{s}}$ becomes

$\Omega_{\mathrm{f}}=R_{\mathrm{f}} \tau_{\mathrm{f}}(\lambda+s)+F_{\mathrm{f}} M P G_{\mathrm{f}} \sqrt{\lambda+s}$

$\Omega_{\mathrm{s}}=R_{\mathrm{s}} \tau_{\mathrm{s}}(\lambda+s)+F_{\mathrm{s}} M P G_{\mathrm{s}} \sqrt{\lambda+s}$

with the following set of transport parameters

$\mathrm{MPG}_{\mathrm{f}}=\sqrt{D_{\mathrm{ef}} R_{\mathrm{pf}} \varepsilon_{\mathrm{pf}}}$

$\mathrm{MPG}_{\mathrm{s}}=\sqrt{D_{\mathrm{es}} R_{\mathrm{ps}} \varepsilon_{\mathrm{ps}}}$

The solution is a product of two exponential terms describing the simultaneous contributions of the two systems in retarding solute transport in a flow channel. In the preceding solution, $\tau_{\mathrm{f}}$ is the water residence time in the channel. $F_{\mathrm{f}}$ is the ratio of flow-wetted surface of rock matrix adjacent to the channel to the water flow rate in the same channel, and gives a measure of relative importance of advection in the channel and diffusion through the rock matrix. Similarly, the parameter group $N$ is the ratio of diffusion rate into the STWZ to the 
Fig. 9 Solute transport in a channel with diffusion into the stagnant water zone (STWZ) and its adjacent rock matrix, after Shahkarami et al. (2015)

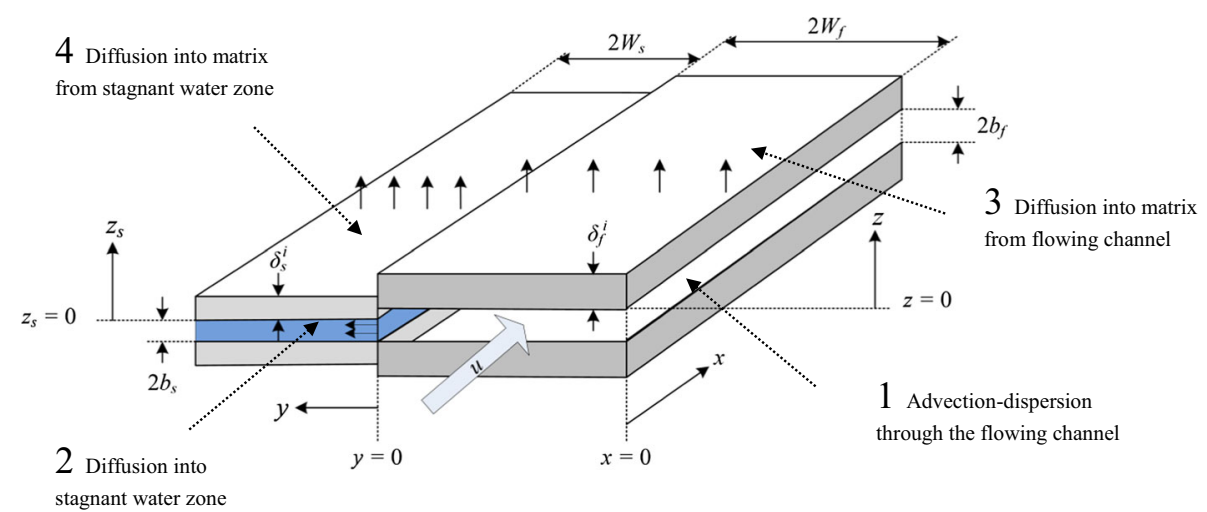

advection rate in the channel and gives a measure of the tendency of solutes to depart from the flow channel toward the STWZ. In fact, these two parameters together with the material property group, MPG, are the key entities that determine the fate of sorbing solute in channels. Nevertheless, due to the existence of spatial heterogeneities, channels in fractures can have very different properties and flow conditions; therefore, the individual channels provide local $F_{\mathrm{f}}$ and $N$ values that result in varying degree of matrix diffusion effect in different channels.

However, as discussed earlier, the flow condition cannot, in most cases, be adequately approximated by the continuum model; thus, the effect of heterogeneities is instead interpreted by introducing the calibrated field-scale matrix diffusion coefficient. A representative example of this approach is the recent survey study carried out by Zhou et al. (2007) which, for ease of reference, is referred to as "the Zhou study" in the remainder of this paper. Zhou et al. (2007) compiled 40 field tracer tests to explore the enhanced effect of matrix diffusion in fractured sites. The calibration results were either collected from the references cited in the Zhou study or were calculated using the continuum model. In other words, fractured media were treated as single discrete fracture-matrix systems with constant fracture aperture and constant fluid velocity where transport parameters represented a lumped effect of spatial heterogeneities on tracer transport. The calibrated transport parameters were

$T_{0}=\frac{L}{U}$
$\mathrm{Pe}=\frac{U L}{D_{L}}$

$A=\frac{\sqrt{D_{\mathrm{ef}} R_{\mathrm{pf}} \varepsilon_{\mathrm{pf}}}}{b}$

$T_{0}$ is the calibrated mean residence time representing the different residence times in multitude of flow paths caused by aperture variability in the fracture plane. The calibrated Peclet number represents the dispersion in the system caused by the velocity difference within and between different channels. The calibrated group A is called the diffusive mass-transfer parameter and represents the lumped effect of different complex diffusive processes. Zhou et al. (2007) concluded that the field-scale matrix diffusion coefficients calculated through the calibrated $A$-value, exhibited much larger values compared to the laboratory-scale matrix diffusion coefficients.

One major drawback of this analysis is that the survey is biased toward finding a larger matrix diffusion coefficient, instead of focusing on the underlying physics responsible for the enhanced diffusive mass exchange. A striking example of this situation is the enhanced $A$-value reported for one case study in Neretnieks (2002) where a STWZ exists in the fracture plane. The STWZ, in fact, increases the available surface area for solute diffusive mass exchange and results in a larger $A$-value, i.e., 77 times larger. This effect, however, is interpreted as the enhanced field-scale matrix diffusion coefficient in the Zhou study.

Another problem that may undermine the conclusion made in the Zhou study is the uncertainty involved in the primary focus of the calibration procedures. In a calibration, very different fitted values are often expected for $T_{0}$, Pe and $A$ if the focus shifts from the rising limb to the middle part (the peak arrival time), or further to the tailing. This makes the calibrated results vulnerable to the objectives of the fittings - for example, in a tracer test where the tracer BTC is dominated by the matrix diffusion and thus exhibits long tailing, a very small $\mathrm{Pe}$ value is often expected to obtain a good fit to the test data. The physical meaning and credibility of this small Pe number, as a measure for the dispersion solely caused by velocity variation, is therefore questionable, knowing that the tailed distribution is dominated by the matrix diffusion. It should also be noted that, in this approach, it is likely that good fits can be achieved with different sets of fitting parameters, which also gives rise to the concern of the tentative interpretation of experimental BTCs.

The most important shortcoming of this analysis, as discussed in the Zhou study but not properly justified, is the use of $t_{\mathrm{w}} / b$ to estimate the ratio of flow-wetted-surface area, 
FWSA, to the flow rate $(Q)$ in a fractured site. This ratio is a key parameter in estimating the field-scale matrix diffusion coefficient. In practice, however, $Q$ and FWSA are independent from each other and their ratio is independent from $b$ and $t_{\mathrm{w}}$. Given a known hydraulic gradient, the flow field can readily be determined from conductance distribution in the site, as embodied in the CNM. Furthermore, the specific flow-wetted-surface area can be estimated from independent measurement and observations in the fractured media. It is, therefore, expected that choice of the aperture value would not change the $F_{\mathrm{f}}$ ratio and thus would not alter the matrix diffusion effect, provided the transport is dominated by the matrix diffusion. In the Zhou study, however, the $F_{\mathrm{f}}$ in a fracture-matrix system is represented by $t_{\mathrm{w}} / b$ where $b$ is embedded in the $A$ parameter, which makes the $F_{\mathrm{f}}$ ratio vulnerable to the aperture estimation error and to the calibrated $t_{\mathrm{w}}$ with its inherent estimation biases, as previously mentioned. These concerns are addressed in the CNM, where the $F_{\mathrm{f}}$ ratio is considered as an independent parameter in individual channels that can be estimated from the specific flowwetted-surface area of the fractured site and flow distribution in the channel network.

\section{The CNM and how the retarding mechanisms are treated}

In the CNM, when the channel transmissivity distribution is known, the flow distribution is obtained by solving the flow equations with given boundary conditions. The volume of the channels can also be determined by the cubic law or modification thereof. Solute can therefore migrate along different transport paths between injection and collection points in the rock volume where velocity can vary between and within the constituent channels. Thus, the solute retarding mechanisms, including the diffusion process into the rock matrix, are controlled by the local values of transport parameters in every channel, and the solute particles that traverse different pathways will experience varying degrees of retardation. The net effect can ultimately be captured by collecting and mixing the streams coming out of the different transport paths to the observation location. In this way, it is likely to capture the joint effect of the retarding mechanisms and velocity dispersion on the solute transport behavior.

Furthermore, the CNM can account for the effect of STWZs situated adjacent to the flow channels. Once considered, the STWZs can provide additional surface for matrix diffusion in individual channels and thereby enhance the matrix-channel interaction. Additionally, in the CNM, every channel can provide a new surface area to the STWZ, which can considerably increase the surface area over which solute can diffuse into the rock matrix and, therefore, increase the matrix diffusion impact. It is worth emphasizing that the impact of the STWZ and its adjacent rock matrix is shown to increase with transport distance, and their joint retarding effect can largely influence solute distribution within the rock volume, given the solute travel time is sufficiently large (Shahkarami et al. 2015, 2016). Furthermore, in narrow channels the matrix diffusion would increasingly become radial as the penetration length becomes larger than the channel width, which will also enhance the matrix diffusion rate. The CNM also allows to introduce heterogeneities in the rock matrix (Mahmoudzadeh et al. 2013) as well as to incorporate objects including stochastic and deterministic fractures, fracture zones, tunnels, etc. that exist in the fractured rocks. The latter is done in a simplified manner in CHAN3D by locating the channels enclosed by the objects and modifying their flow and transport properties, i.e., conductance and FWSA, respectively (Liu et al. 2010).

In light of the preceding discussion, it is believed that the CNM can provide a simple, yet powerful, platform to capture the impacts of spatial heterogeneities in fractured media including (1) heterogeneities of the flow distribution and transport parameters (2) heterogeneities in the rock matrix, i.e., multi-layer diffusive mass transfer, and (3) heterogeneities in the fracture planes, i.e., diffusion into the STWZs. When it comes to the matrix-diffusion effect, these heterogeneities contribute to varying degree of interactions between the channels and the matrix.

\section{The STT-1 test: simulation of a sorbing nuclide}

In what follows, to support the concern regarding the credibility of the calibration results and also to highlight the differences between the continuum model and the CNM, the STT-1 test is simulated (see section 'Use of the Taylor dispersion and continuum model in the STT-1 test'). However, this time the model is conducted with the sorbing tracer Caesium-137, ${ }^{137} \mathrm{Cs}$, with effective diffusion coefficient $\mathrm{De}_{\mathrm{Cs}}=4.25 \times$ $10^{-14} \mathrm{~m}^{2} / \mathrm{s}$ and sorption coefficient $K_{\mathrm{d}}=0.29 \mathrm{~m}^{3} / \mathrm{kg}$. No diffusion into the STWZs is assumed here because its retarding effect is shown to be negligible over the short time-scale of the test (Shahkarami et al. 2016).

Initially, the same procedure as described in the Zhou study is followed, where the continuum model is employed to find the calibrated $t_{\mathrm{w}}$, Pe and the scale factor, SF, defined as the ratio of the would-be field-scale matrix diffusion coefficient, De*, to the effective diffusion coefficient De.

To find the De*, it is also necessary to know the average aperture size $(2 b)$ of the single fracture-matrix system, as envisaged in the continuum model, Eq. (18). In section 'Use of the Taylor dispersion and continuum model in the STT-1 test', the average aperture of the ensemble of the channels is estimated to be $2 b_{\text {avg }}=0.05 \mathrm{~mm}$. This value is used in the following analysis. However, it should be noted that if the test conditions and rock volume are not well characterized, several uncertainties may arise when one tries to estimate the aperture 
Fig. 10 Continuum modelpredicted results and experimental BTC for ${ }^{137} \mathrm{Cs}$

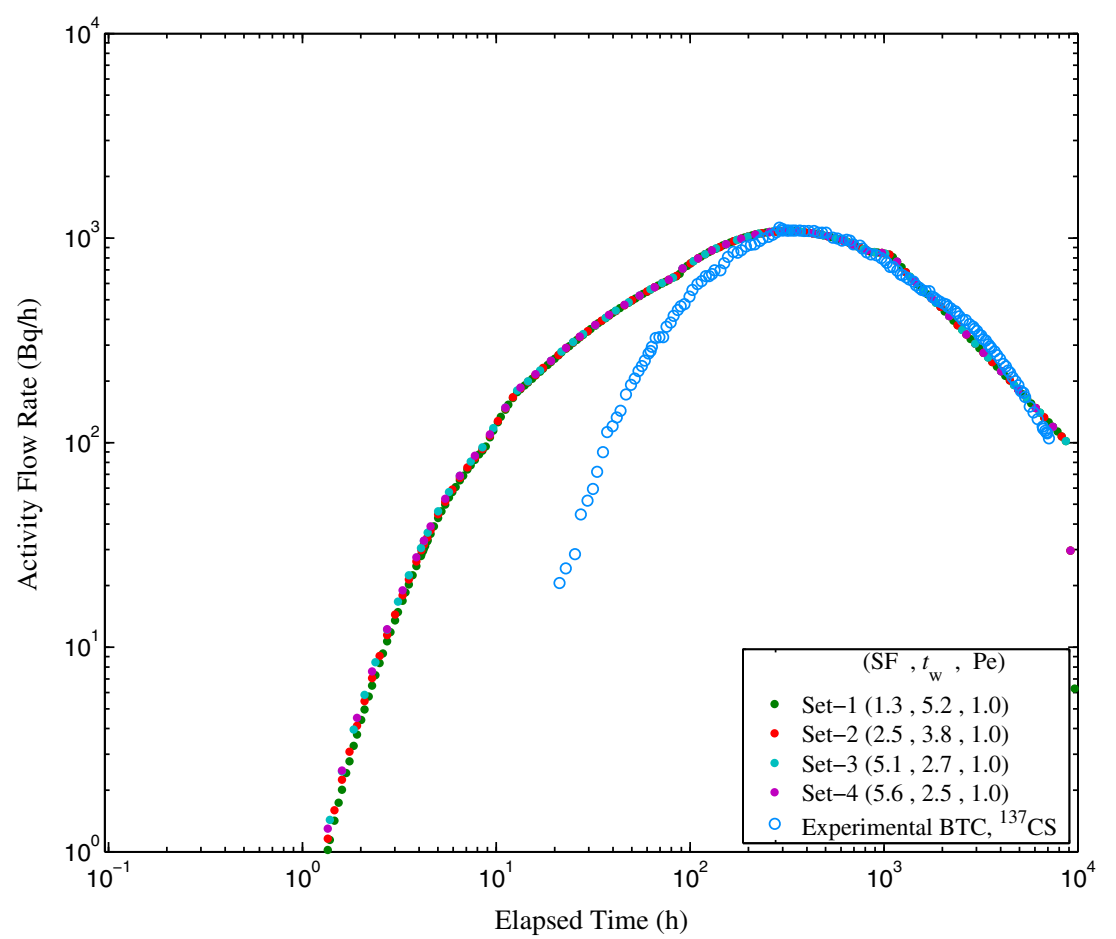

size, either from the hydraulic data or from the nonsorbing tracer test results, which may make the estimated De* value vulnerable to the aperture estimation error.

The experimental BTC and predicted results for ${ }^{137} \mathrm{Cs}$ are shown in Fig. 10. The continuum model predicted results are obtained for four different sets of the calibrated parameters $t_{\mathrm{w}}$, $\mathrm{Pe}$, and SF, as shown in the legend of the figure. The calibration is made to find the best fit to the late-time behavior of the experimental BTC, i.e., the tailing. It can be seen that the SF can take different values, ranging from 1.3 to 5.6, corresponding to different values of $t_{\mathrm{w}}$, which indicates that when the continuum model is employed, a larger value for the matrix diffusion coefficient is, in general, required to explain the observed tailing in the BTC. However, in the meantime, it introduces a potential for bias in selecting the model parameters since all the four sets of parameters can be used to produce the model-predicted BTC.

Furthermore, in all the parameter sets, the calibrated Peclet numbers exhibit smaller values, $\mathrm{Pe}=1$, compared to the $\mathrm{Pe}=$ 5 found from the preceding test with the nonsorbing tracers in section 'Use of the Taylor dispersion and continuum model in the STT-1 test'. Despite the error involved in applying the $\mathrm{ADE}$ to find the $\mathrm{Pe}=5$, it is yet supposed to be a better measure for the dispersion solely caused by the velocity variation in the system. The inconsistency between the calibrated $\mathrm{Pe}=1$ and $\mathrm{Pe}=5$ together with the unknown physical explanation behind this inconsistency make the calibrated parameters $t_{\mathrm{w}}$, $\mathrm{Pe}$, and SF questionable. Not to mention that these values are valid only under the test conditions and should not be used to, e.g., extend the model results to other distances or flow rates (Liu et al. 2017).

The situation is, however, quite different when the CNM is employed to describe the tracer transport. The swarm of points in Fig. 11 represents the predicted BTCs for 100 realizations and the solid line represents the corresponding mean BTC.

In these realizations, no Fickian-type dispersion is invoked and only advection and matrix diffusion are active in channels. It can be seen that the simulation result fairly well agrees with the experimental BTC, considering that no parameter fitting is made. In other words, the CNM allows predicting the tracer BTC by using the laboratory-measured diffusion and sorption parameters. This became possible because the CNM provides a physically meaningful framework to incorporate tracer transport within fast and slow pathways. In this way, the tracer particles can experience local values of transport parameters in every channel, for which their properties are stochastically, or deterministically, assigned to represent the impact of heterogeneities that exist in the rock volume. Regarding the matrix diffusion effect, this feature is illustrated in Fig. 12, where the histogram distribution of $F_{\mathrm{f}}$ values that the tracer particles experience in their pathways is shown.

The net effect can then be captured by collecting and mixing the streams coming out of the different transport paths. In Figs. 11 and 13, it can be seen that the tailing slope in the mean of the resulted BTCs is slightly smaller than $-3 / 2$, the well-known slope for matrix diffusion effect in a single channel. This highlights the role of velocity dispersion, caused by heterogeneities, on the late-time behavior of the tracer. To 
Fig. 11 Simulated and experimental BTCs for ${ }^{137} \mathrm{Cs}$

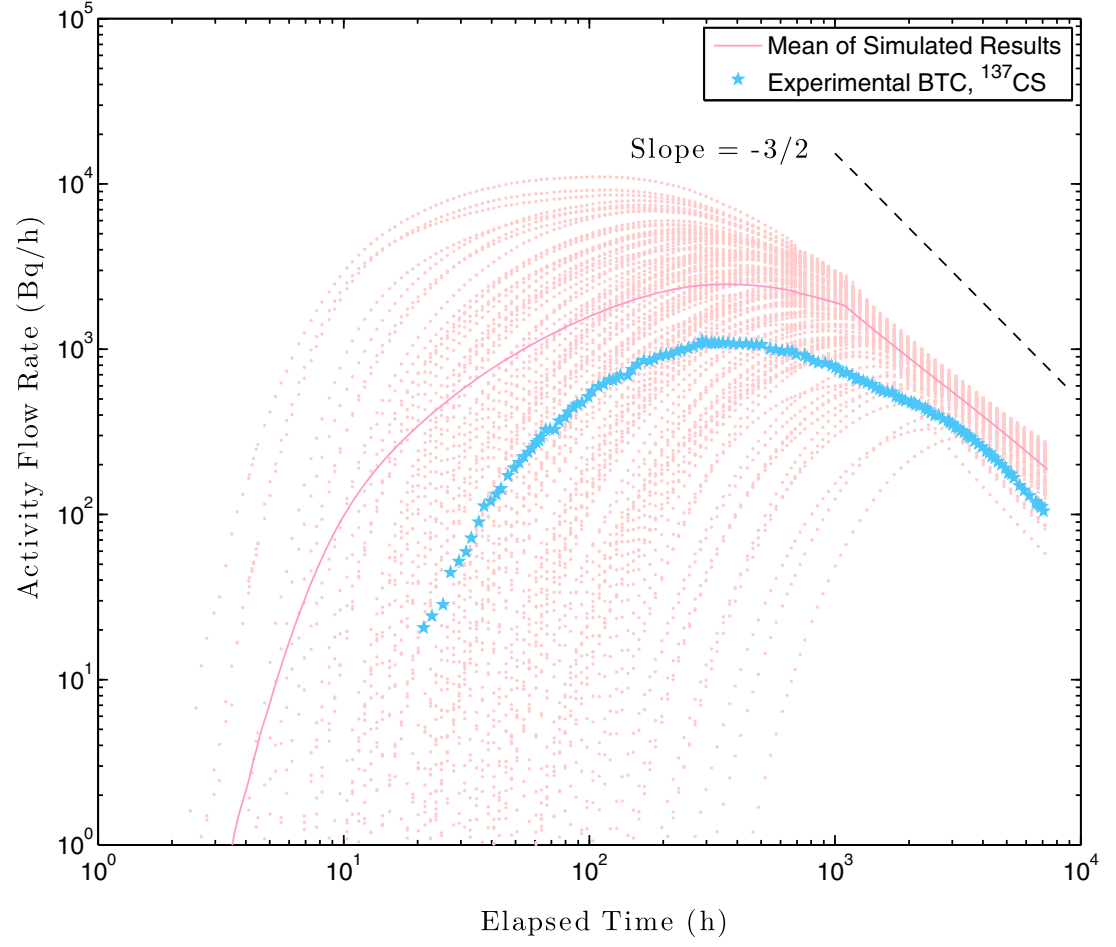

make it clearer, one may consider a situation where it is assumed that all the channels in the network have similar transmissivities, i.e., $\sigma=0$; thus, the effect of velocity dispersion is neglected in the network. In Fig. 13, the simulated BTC obtained under this condition is plotted and compared to the mean of the resulted BTCs with $\sigma=0.97$.
It is evident from the plots that there is a significant difference between the two conditions. The BTC with $\sigma=0$ now exhibits a tailing slope which is consistent with $-3 / 2$, expected solely due to the matrix diffusion effect. Furthermore, the effect of retarding mechanisms is largely underestimated owing to neglecting the impact of velocity distribution in the
Fig. $12 F_{\mathrm{f}}$ histogram distributions for one channel-network realization (number of injected particles $=1,000)$

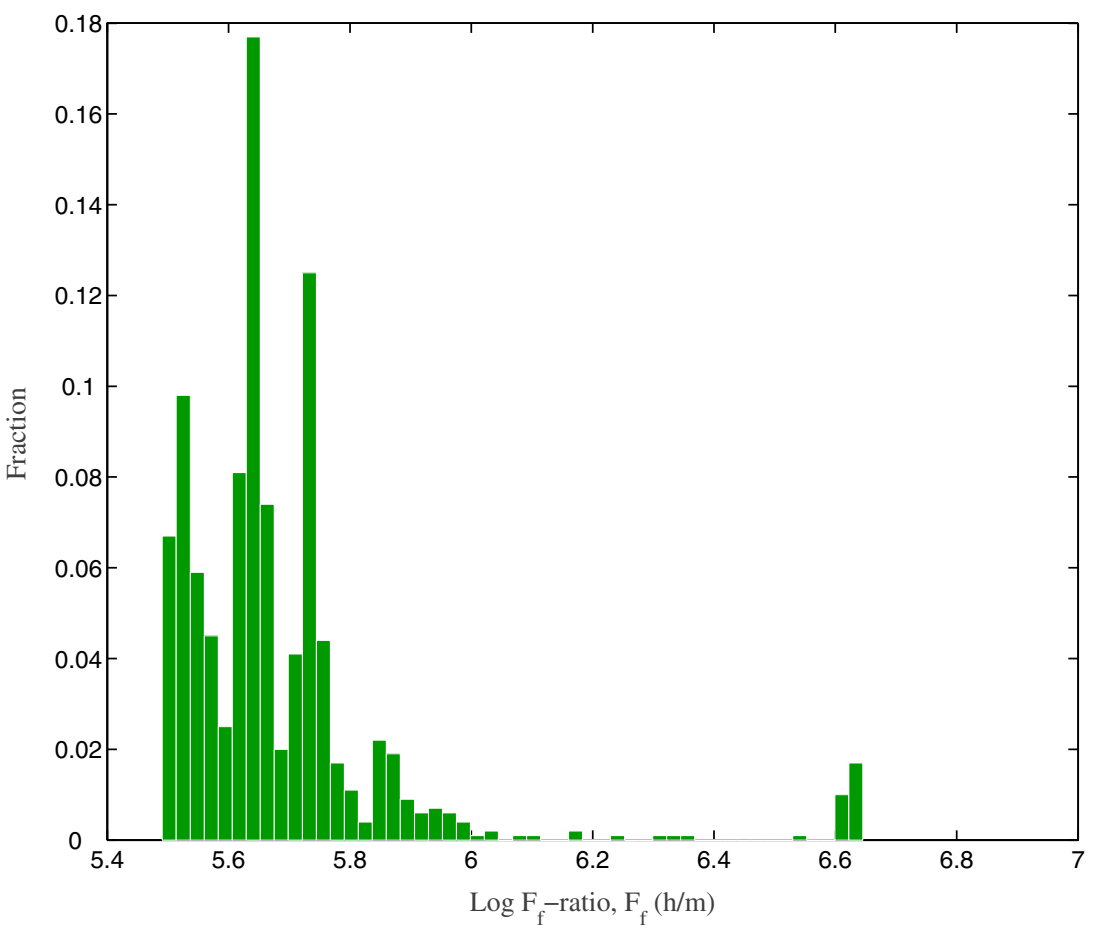


Fig. 13 Effect of velocity distribution on ${ }^{137} \mathrm{Cs}$ BTCs

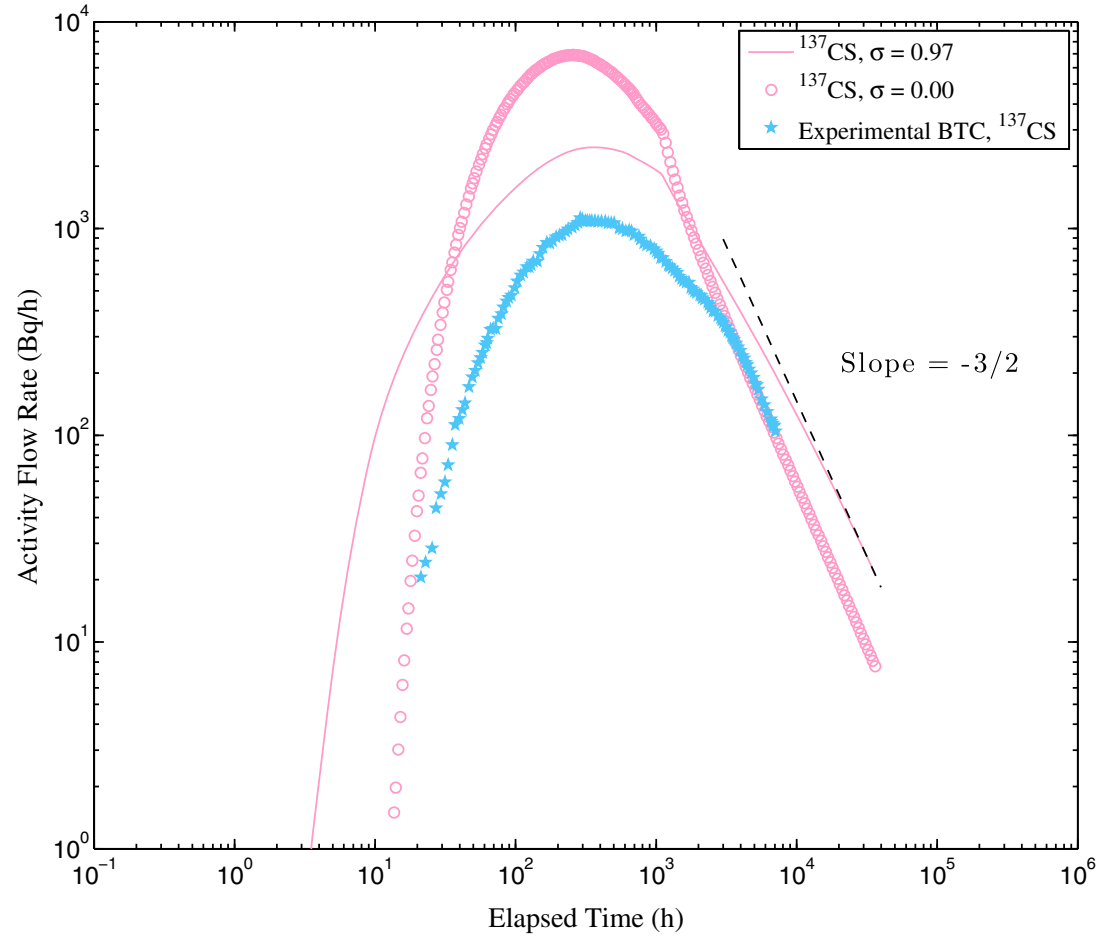

network. This finding, again, highlights the importance of the detailed flow-field information for the proper description of tracer transport behavior in fractured rocks.

It is important to note that, although mathematically feasible, the authors have no intention to calibrate the CNM parameters to find the best fit of the experimental BTCs. The authors, on the other hand, would like to draw the attention to the relevance of the CNM to address the actual behavior of solute transport in fractured rocks. The CNM provides a convenient framework to introduce the heterogeneities that exist in a fractured system and allows for the prediction of the closeto-the-real behavior of solute transport in fractured rocks, given the laboratory-measured transport properties.

The inconsistency between the simulated and experimental BTCs may partly be attributed to the uncertainties inherent in obtaining the hydraulic data, sorption coefficient, and the effective diffusion coefficient in the laboratory. Another possible explanation might be the assumption that the complete mixing theory is valid within the channel intersections. If not, particles may be distributed in a different manner than when mixing is complete and form different pathways with fewer or greater numbers of channels. The overall retarding effect of the system may, therefore, change.

\section{Discussion and conclusions}

This study is intended to improve understanding of solute transport in fractured rocks. The various physical mechanisms underlying the transport of solute are discussed and, in light of the evidence reviewed in this paper, it is suggested that it is feasible and more physically meaningful to describe flow and transport in fractured rocks as taking place in a 3D network of stochastic channels.

In this paper, this concern is raised again that the use of the continuum model often gives rise to uncertainty and confusion in interpretation of field-scale tracer experiments - mostly because it is required to introduce effective entities, i.e., macro dispersion coefficient and field-scale matrix diffusion coefficient, to capture the effect of spatial heterogeneities. These entities are thus believed to be vulnerable to the heterogeneities in the system and should not be used to, e.g., extend the model results to other distances, flow rates or directions. It is also shown that estimation of these entities is subject to sometimes large theoretical and physical uncertainties and errors.

To address the question of when it is physically appropriate to utilize the concept of macro dispersion, a simple theory is presented in this paper to find the time threshold beyond which the continuum model is applicable, and when the ADE is valid in a tapered channel. The subsequent analysis, aimed to find the proper way to visualize the solute transport, is made under natural- and forced-gradient flow conditions. The results reveal that, while under the natural-gradient condition, the ADE might be valid in individual channels; spreading of the tracer pulse under the forced-gradient condition, as in tracer test experiments, is mostly dominated by advective mechanisms and velocity dispersion. Therefore, the use of the $\mathrm{ADE}$ and the macro dispersion coefficient can be 
misleading when applied to interpret the experimental BTCs. Furthermore, the results suggest that it is feasible to visualize flow and transport as taking place in many different pathways that seldom mix with other pathways.

The paper goes on to criticize the idea behind introducing the field-scale matrix diffusion coefficient in fractured rocks. Aside from the point that the validity of the continuum model is often questionable under tracer test conditions, the concerns in this part are mainly directed toward the uncertainties and potential bias involved in finding the model calibrated parameters, and hence the field-scale matrix diffusion coefficient. These concerns are highlighted through the predictive modeling of a sorbing tracer in the well-defined STT- 1 tracer test.

In light of the results of the analysis performed in this paper, the authors believe that the overwhelming efforts directed toward defining the effective entities to address the transport problem in fractured rock are misplaced for the detailed understanding of hydraulic behavior and solute transport mechanisms. If available, this information can provide a reliable basis upon which one can build a realistic framework to explain the solute transport behavior in fractured rocks, as incorporated in the channel network concept, $\mathrm{CN}$-concept.

It is shown that the $\mathrm{CN}$-concept, and its computer implementation, i.e. channel network model, CNM, can accommodate many important physical mechanisms underlying the natural behavior of solute transport in fractured rocks, when their impacts otherwise would be interpreted as effective lumped entities. Other strong features of the $\mathrm{CN}$-concept are also discussed in this paper; when applicable, their impacts can largely influence the solute transport behavior in fractured rocks.

Nevertheless, it should be stressed that the analyses made in this study are limited to a fixed distance between the injection and observation points. Unfortunately, the authors could not find experimental data on a well-characterized tracer test in fractured crystalline rocks that was performed at different distances from the injection point. At longer distances in fractured rocks when (1) very long pathways exist in the system and (2) long gaps exist between the pathways, it is required to take into account the correlation between the channel length and channel conductance. Thus, longer channels may result in higher conductance, which is expected to be the case in rocks with a wide distribution of fracture sizes in which the transmissivities of the channels are expected to be larger in larger fractures. This situation can be addressed in the CNM by introducing fracture zones or deterministic fractures; however, introducing the correlation length can help to better visualize the solute transport via the CNM. Otherwise, if the fracture zone data are not available, assigning channel conductance randomly may result in a channel network where slow-flow channels block fast-flow channels and suppress the system connectivity. Future research should therefore focus on introducing the conductance-length correlation in the CNM.
It may be noted that no reliable model is currently available to describe the BTC in a tapered channel for the transition region, i.e., $0.02<T<0.6$. Furthermore, when $T<0.02$, no mixing takes place between the streamlines, and the parabolic velocity distribution in the channel gives a very wide, if not infinite, residence time distribution in the channel. In the stochastic simulations made in this paper, however, the flow is assumed to be uniform, under the aforementioned two conditions.

It may also be noted that in the tapered channel, it is assumed that no simultaneous matrix diffusion from the streamlines takes place with diffusion between them. Instead, the matrix diffusion is modeled as if it takes place to/from the already mixed streamlines. This is an approximation.

Acknowledgements The authors gratefully acknowledge the encouragement and financial support of the Swedish Nuclear Fuel and Waste Management Company (SKB).

\section{Appendix: the validity range of the Taylor dispersion approximation}

In this appendix, a simple practical theory is presented to find the time threshold beyond which the advection-dispersion equation is applicable in a tapered channel, as schematically shown in Fig. 7. Due to the geometric symmetry, it is only needed to study the right half of the channel. Consider a case where a long-tapered channel with width $2 W_{\mathrm{f}}$ initially with concentration 0 is subjected to a concentration $C_{0}$ at $\mathrm{y}=0$ for $t>0$. It is of interest to explore how the concentration in the channel evolves over time, which is similar to exploring how fast the concentration between the most distant streamlines in the parabolic profile across the channel width would even out with transit time in the flowing channel.

Given $\left(r=W_{\mathrm{f}}-y\right)$, the mass balance equation becomes

$\frac{\partial c(r, t)}{\partial t}=\frac{D_{\mathrm{w}}}{r} \frac{\partial}{\partial r}\left(r \frac{\partial c}{\partial r}\right)$

with the boundary conditions given by

$c\left(r=W_{\mathrm{f}}\right)=C_{0}$

$\left.\frac{\partial c}{\partial r}\right|_{r=0}=0$

The solution to the purely radial diffusion equation, with the given initial and boundary conditions is (Carslaw and Jaeger 1959)

$\frac{c(r, t)}{C_{0}}=\frac{2}{R} \sum_{n=1}^{\infty} \frac{J_{0}\left(\lambda_{\mathrm{n}} r\right)}{\lambda_{\mathrm{n}} J_{1}\left(\lambda_{\mathrm{n}} W_{\mathrm{f}}\right)} \exp \left(-D_{\mathrm{w}} \lambda_{\mathrm{n}}^{2} t\right)$ 
Fig. 14 Concentration evolution at the tube center as a function of the dimensionless time $T$

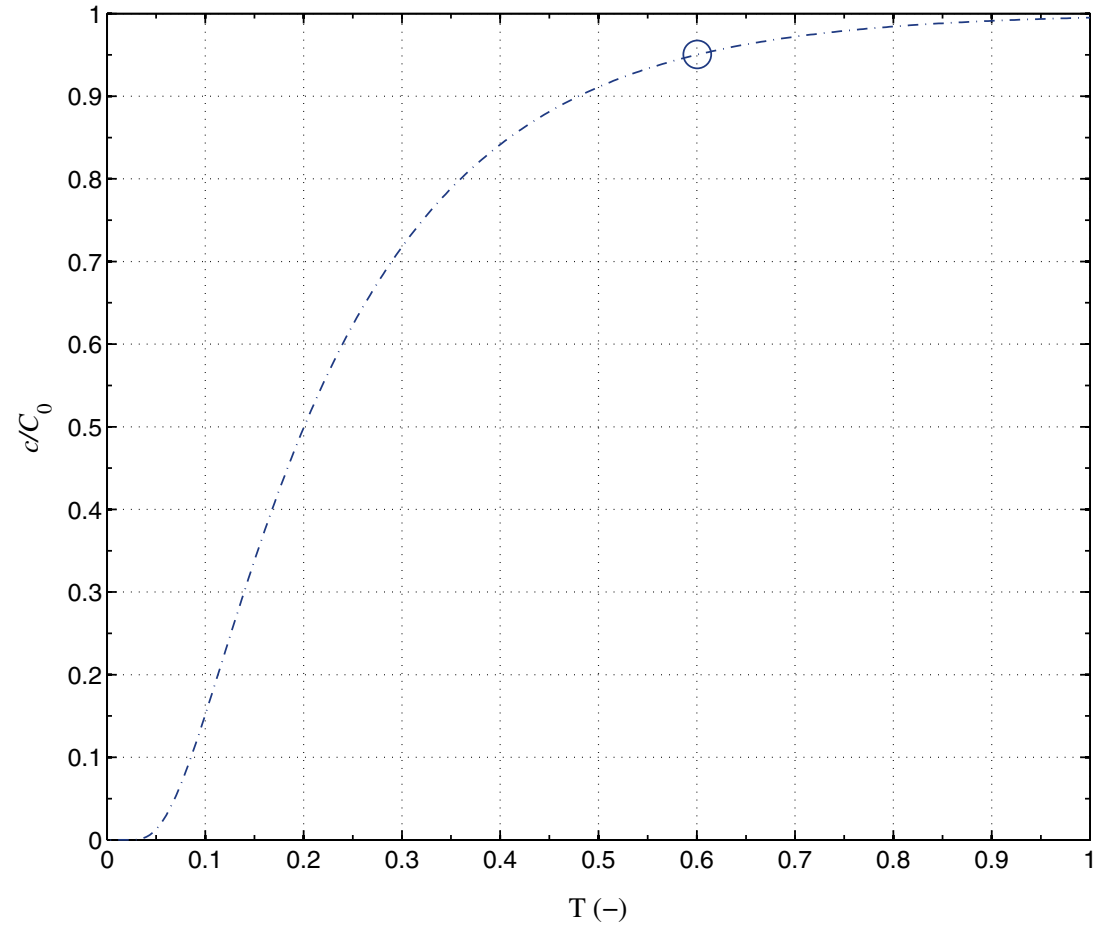

$J_{0}$ is the Bessel function of the first kind, and $\lambda_{\mathrm{n}}$ are eigenvalues of this problem and are thus given as the roots of

$J_{0}\left(\lambda_{\mathrm{n}} W_{\mathrm{f}}\right)=0$

The dimensionless diffusion time, $T$, is also defined as:

$T=\frac{D_{\mathrm{w}} t}{W_{\mathrm{f}}^{2}}$

Figure 14 shows how the mean concentration in the channel increases with the dimensionless time $T$. It can be seen that even over short times, noticeable evening out of the concentration in the channel will take place between streamlines; however, a very long time is needed to fully even out the concentration. At $T=0.6$ the mean concentration still deviates by $5 \%$ and at $T=0.9$ it deviates by $1 \%$.

For the purposes of the present study, the region $0.02<T<$ 0.6 is considered to be the transition region between the two extreme conditions where dispersion is dominated by flow rate differences and Taylor dispersion. The result of this simple analysis is comparable to that obtained by Wang et al. (2012).

Open Access This article is distributed under the terms of the Creative Commons Attribution 4.0 International License (http:// creativecommons.org/licenses/by/4.0/), which permits unrestricted use, distribution, and reproduction in any medium, provided you give appropriate credit to the original author(s) and the source, provide a link to the Creative Commons license, and indicate if changes were made.

\section{References}

Aris R (1956) On the dispersion of a solute in a fluid flowing through a tube. Proc Roy Soc Lond A 235:67-77

Becker MW, Shapiro AM (2000) Tracer transport in fractured crystalline rock: evidence of nondiffusive breakthrough tailing. Water Resour Res 36(7):1677-1686

Becker MW, Shapiro AM (2003) Interpreting tracer breakthrough tailing from different forced-gradient tracer experiment configurations in fractured bedrock. Water Resour Res 39(1):1024. https://doi.org/ 10.1029/2001WR001190.

Berkowitz B, Bear J, Braester C (1988) Continuum models for contaminant transport in fractured porous formations. Water Resour Res 24(8):1225-1236

Berkowitz B, Scher H (1997) Anomalous transport in random fracture networks. Phys Rev Lett 79(20):4038-4041

Berkowitz B, Klafter J, Metzler R, Scher H (2002) Physical pictures of transport in heterogeneous media: advection-dispersion, random-walk, and fractional derivative formulations. Water Resour Res 38(10):1191. https://doi.org/10.1029/ 2001WR001030.

Carslaw HS, Jaeger JC (1959) Conduction of heat in solids. Clarendon, Oxford

Carrera J, Sánchez-Vila X, Benet I, Medina A, Galarza G, Guimerà J (1998) On matrix diffusion: formulations, solution methods and qualitative effects. Hydrogeol J 6(1):178-190

Cushman JH, Ginn TR (2000) Fractional advection-dispersion equation: a classical mass balance with convolution-Fickian flux. Water Resour Res 36(12):3763-3766

Delgado JMPQ (2006) A critical review of dispersion in packed beds. Heat Mass Transf 42(4):279-310

Elert M, Svensson H (2001) Evaluation of modelling of the TRUE-1 radially converging tests with sorbing tracers: the Äspö Task Force on Modelling of Groundwater Flow and Transport of Solutes, tasks 4E and 4F. Technical report TR-01-12, SKB, Stockholm 
Gao G, Zhan H, Feng S, Huang G, Mao X (2009) Comparison of alternative models for simulating anomalous solute transport in a large heterogeneous soil column. J Hydrol 377(3):391-404

Gelhar LW, Welty C, Rehfeldt KR (1992) A critical review of data on field-scale dispersion in aquifers. Water Resour Res 28(7):19551974

Grisak GE, Pickens JF (1980) Solute transport through fractured media: 1. the effect of matrix diffusion. Water Resour Res 16(4):719-730

Guerin FPM, Billaux DM (1994) On the relationship between connectivity and the continuum approximation in fracture-flow and transport modelling. Appl Hydrogeol 2(3):24-31

Haggerty R, Gorelick SM (1995) Multiple-rate mass transfer for modeling diffusion and surface reactions in media with pore-scale heterogeneity. Water Resour Res 31:2383-2400

Haggerty R, McKenna SA, Meigs LC (2000) On the late-time behavior of tracer test breakthrough curves. Water Resour Res 36(12):34673479

Hodgkinson DP, Maul PR (1988) 1-D modelling of radionuclide migration through permeable and fractured rock for arbitrary length decay chain using numerical inversion of Laplace transforms. Ann Nucl Energy 15(4):175-189

Huysmans M, Dassargues A (2005) Review of the use of Péclet numbers to determine the relative importance of advection and diffusion in low permeability environments. Hydrogeol J 13(5-6):895-904

Jardine PM, Sanford WE, Gwo JP, Reedy OC, Hicks DS, Riggs JS, Bailey WB (1999) Quantifying diffusive mass transfer in fractured shale bedrock. Water Resour Res 35(7):2015-2030

Kang PK, Dentz M, Le Borgne T, Juanes R (2015) Anomalous transport on regular fracture networks: impact of conductivity heterogeneity and mixing at fracture intersections. Phys Rev E 92(2):022148

Liu HH, Haukwa CB, Ahlers CF, Bodvarsson GS, Flint AL, Guertal WB (2003) Modeling flow and transport in unsaturated fractured rock: an evaluation of the continuum approach. J Contam Hydrol 62:173188

Liu HH, Zhang YQ, Zhou Q, Molz FJ (2007) An interpretation of potential scale dependence of the effective matrix diffusion coefficient. J Contam Hydrol 90(1):41-57

Liu L, Moreno L, Neretnieks I, Gylling B (2010) A safety assessment approach using coupled NEAR3D and CHAN3D: Forsmark. Report R-10-69. SKB, Stockholm, Sweden

Liu L, Neretnieks I, Shahkarami P, Meng S, Moreno L (2017) Solute transport along a single fracture in a porous rock: a simple analytical solution and its extension for modeling velocity dispersion. Hydrogeol J 26(1):297-320

Long JCS, Remer JS, Wilson CR, Witherspoon PA (1982) Porous media equivalents for networks of discontinuous fractures. Water Resour Res 18(3):645-658

Mahmoudzadeh B, Liu L, Moreno L, Neretnieks I (2013) Solute transport in fractured rocks with stagnant water zone and rock matrix composed of different geological layers: model development and simulations. Water Resour Res 49(3):1709-1727

Małoszewski P, Zuber A (1985) On the theory of tracer experiments in fissured rocks with a porous matrix. J Hydrol 79(3-4):333-358

Maloszewski P, Zuber A (1990) Mathematical modeling of tracer behavior in short-term experiments in fissured rocks. Water Resour Res 26(7):1517-1528

Maloszewski P, Zuber A (1993) Tracer experiments in fractured rocks: matrix diffusion and the validity of models. Water Resour Res 29(8): 2723-2735

Matheron G, de Marsily G (1980) Is transport in porous media always diffusive? A counterexample. Water Resour Res 16(5):901-917. https://doi.org/10.1029/WR016i005p00901

McKay LD, Gillham RW, Cherry JA (1993) Field experiments in a fractured clay till: 2 . solute and colloid transport. Water Resour Res 29(12):3879-3890
McKenna SA, Meigs LC (2000) On the late-time behavior of tracer test breakthrough curves. Water Resour Res 36(12):3467-3479

Moench AF (1995) Convergent radial dispersion in a double-porosity aquifer with fracture skin: analytical solution and application to a field experiment in fractured chalk. Water Resour Res 31(8):18231835

Moreno L, Neretnieks I (1993) Fluid flow and solute transport in a network of channels. J Contam Hydrol 14:163-192

Moreno L, Tsang CF, Tsang Y, Neretnieks I (1990) Some anomalous features of flow and solute transport arising from fracture aperture variability. Water Resour Res 26(10):2377-2391

Neretnieks I (1983) A note on fracture dispersion mechanisms in the ground. Water Resour Res 19(2):365-370. https://doi.org/10.1029/ WR019i002p00364.

Neretnieks I (2002) A stochastic multi-channel model for solute transport: analysis of tracer tests in fractured rock. J Contam Hydrol 55(3): $175-211$

Neretnieks I (2005) Channeling with diffusion into stagnant water and into a matrix in series. Water Resour Res 42:W11418

Neretnieks I (2017) Solute transport in channel networks with radial diffusion from channels in a porous rock matrix. SKB report R-15-02, SKB, Stockholm

Neretnieks I, Moreno L (2003) Prediction of some in situ tracer tests with sorbing tracers using independent data. J Contam Hydrol 61:351360

Neretnieks I, Eriksen T, Tähtinen P (1982) Tracer movement in a single fissure in granitic rock: some experimental results and their interpretation. Water Resour Res 18(4):849-858

Neuman SP, Winter CL, Newman CM (1987) Stochastic theory of fieldscale Fickian dispersion in anisotropic porous media. Water Resour Res 23(3):453-466

Ohlsson Y, Neretnieks I (1995) Literature survey of matrix diffusion theory and of experiments and data including natural analogues, Technical report 95-12. SKB, Stockholm

Ohlsson Y, Lofgren M, Neretnieks I (2001) Rock matrix diffusivity determinations by in-situ electrical conductivity measurements. J Contam Hydrol 47:117-125

Pankow JF, Johnson RL, Hewetson JP, Cherry JA (1986) An evaluation of contaminant migration patterns at two waste disposal sites on fractured porous media in terms of the equivalent porous medium (EPM) model. J Contam Hydrol 1(1-2):65-76

Reimus P, Pohll G, Miheve T, Chapman J, Haga M, Lyles B, Kosinski S, Niswonger R, Sanders P (2003) Testing and parameterizing a conceptual model for solute transport in a fractured granite using multiple tracers in a forced-gradient test. Water Resour Res 39(12):1356. https://doi.org/10.1029/2002WR001597.

Shahkarami P, Liu L, Moreno L, Neretnieks I (2015) Radionuclide migration through fractured rock for arbitrary-length decay chain: analytical solution and global sensitivity analysis. J Hydrol 520:448460

Shahkarami P, Liu L, Moreno L, Neretnieks I (2016) The effect of stagnant water zones on retarding radionuclide transport in fractured rocks: an extension to the channel network model. J Hydrol 540: $1122-1135$

Shapiro AM (2001) Effective matrix diffusion in kilometer-scale transport in fractured crystalline rock. Water Resour Res 37(3): $507-522$

Simunek J, van Genuchten MT (2008) Modeling nonequilibrium flow and transport processes using HYDRUS. Vadose Zone J 7:782-797

Skagius K, Neretnieks I (1986) Porosities and diffusivities of some nonsorbing species in crystalline rocks. Water Resour Res 22:389 398

Sudicky EA, Frind EO (1982) Contaminant transport in fractured porous media: analytical solutions for a system of parallel fractures. Water Resour Res 18(6):1634-1642 
Tang DH, Friend EO, Sudicky EA (1981) Contaminant transport in fractured porous media: analytical solution for a single fracture. Water Resour Res 17(3):555-564

Taylor G (1953) Dispersion of soluble matter in solvent flowing slowly through a tube. Proc Roy Soc Lond A 219(1137):186-203

Tsang YW (1995) Study of alternative tracer tests in characterizing transport in fractured rocks. Geophys Res Lett 22(11):1421-1424

Tsang CF, Neretnieks I (1998) Flow channeling in heterogeneous fractured rocks. Rev Geophys 36(2):275-298

Tsang YW, Tsang CF (2001) A particle-tracking method for advective transport in fractures with diffusion into finite matrix blocks. Water Resour Res 37:831-835. https://doi.org/10.1029/2000WR900367
Wang L, Cardenas MB, Deng W, Bennett PC (2012) Theory for dynamic longitudinal dispersion in fractures and rivers with Poiseuille flow. Geophys Res Lett 39(5)

Winberg A, Conterra AB, Andersson P, Hermanson J, Grundteknik G, Byegård J, Cvetkovic V, Birgersson L (2000) Äspö Hard Rock Laboratory. Final report of the first stage of the tracer retention understanding experiments. Technical report TR-00-07, SKB, Stockholm, Sweden

Zhou Q, Liu HH, Molz FJ, Zhang Y, Bodvarsson GS (2007) Field-scale effective matrix diffusion coefficient for fractured rock: results from literature survey. J Contam Hydrol 93(1):161-187 